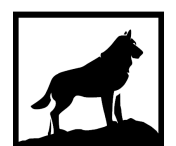

Michigan

Technological

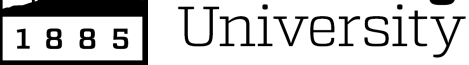

Michigan Technological University

Digital Commons @ Michigan Tech

MISEXPRESSION OF CANCER/TESTIS (CT) GENES IN TUMOR CELLS AND THE POTENTIAL ROLE OF DREAM COMPLEX AND THE RETINOBLASTOMA PROTEIN RB IN SOMA-TO-GERMLINE TRANSFORMATION

SABHA M. ALHEWATI

Michigan Technological University, salhewat@mtu.edu

Copyright 2019 SABHA M. ALHEWATI

Recommended Citation

ALHEWATI, SABHA M., "MISEXPRESSION OF CANCER/TESTIS (CT) GENES IN TUMOR CELLS AND THE POTENTIAL ROLE OF DREAM COMPLEX AND THE RETINOBLASTOMA PROTEIN RB IN SOMA-TOGERMLINE TRANSFORMATION", Open Access Master's Thesis, Michigan Technological University, 2019. https://doi.org/10.37099/mtu.dc.etdr/933

Follow this and additional works at: https://digitalcommons.mtu.edu/etdr Part of the Cancer Biology Commons, and the Cell Biology Commons 


\title{
MISEXPRESSION OF CANCER/TESTIS (CT) GENES IN TUMOR CELLS AND THE POTENTIAL ROLE OF DREAM COMPLEX AND THE RETINOBLASTOMA PROTEIN RB IN SOMA-TO-GERMLINE TRANSFORMATION
}

\author{
By \\ Sabha Salem Alhewati \\ A THESIS \\ Submitted in partial fulfillment of the requirements for the degree of \\ MASTER OF SCIENCE \\ In Biological Sciences
}

MICHIGAN TECHNOLOGICAL UNIVERSITY

2019

(C) 2019 Sabha Alhewati 
This thesis has been approved in partial fulfillment of the requirements for the Degree of MASTER OF SCIENCE in Biological Sciences.

Department of Biological Sciences

Thesis Advisor: $\quad$ Paul Goetsch.

Committee Member: Ebenezer Tumban.

Committee Member: Zhiying Shan.

Department Chair: Chandrashekhar Joshi. 


\section{Table of Contents}

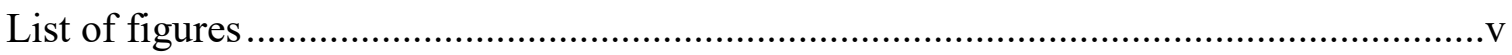

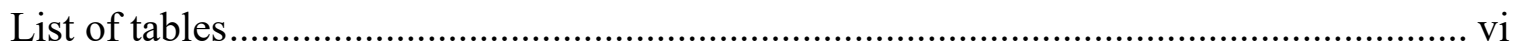

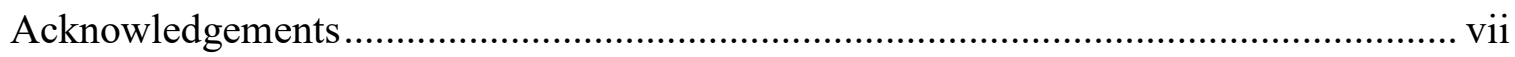

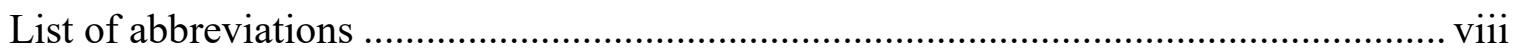

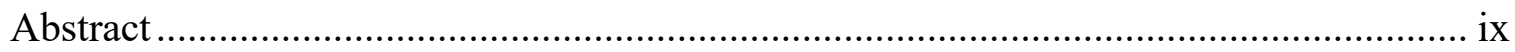

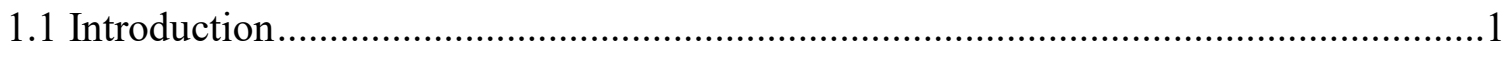

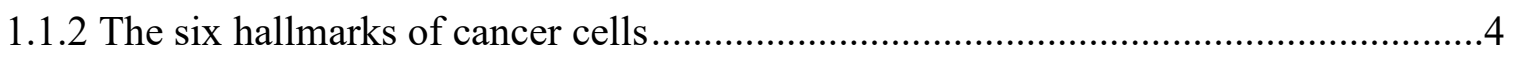

1.1.3 Dysregulation of $\mathrm{pRb}$ is associated with tumor development ................................10

1.1.4 DREAM complex pathway in repression cell cycle genes ......................................13

1.1.5 Downregulation expression of cell cycle genes by p53 …………………….........16

1.1.6 Aberrant expression of cancer/testis (CT) genes in human somatic cells ................19

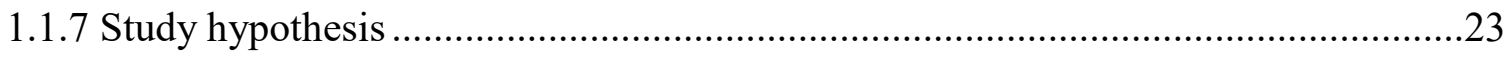

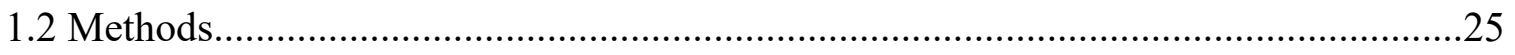

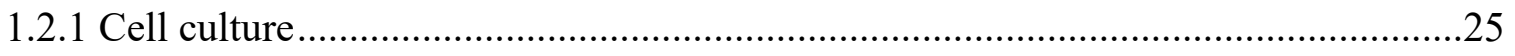

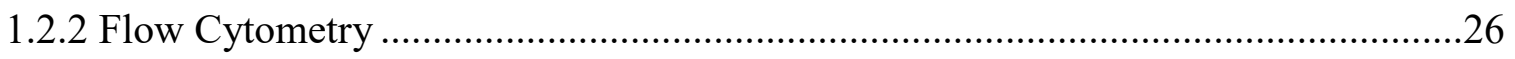

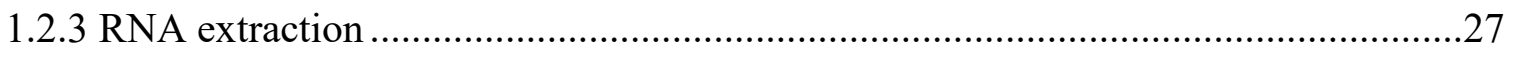

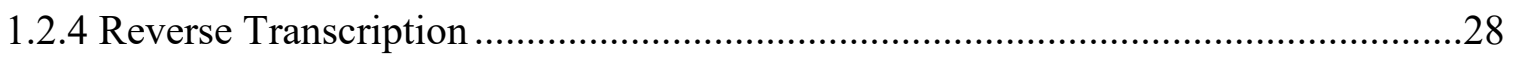

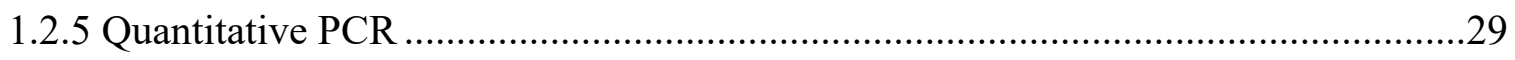

1.2.6 Transfection and luciferase assays .........................................................................30

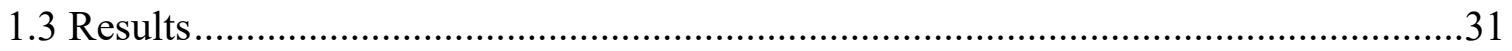

1.3.1 Majority of human cancer cell lines tested arrest their cell cycle in growth-limiting conditions 
1.3.2 Exploring a potential relationship between CT genes expression and loss of

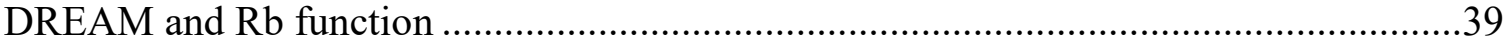

1.3.3 Early cell cycle genes are primarily repressed by both DREAM and Rb.................44

1.3.4 Late cell cycle genes are primarily repressed by DREAM ........................................47

1.3.5 DREAM is functional normally in SW480, and H1299 cell lines............................53

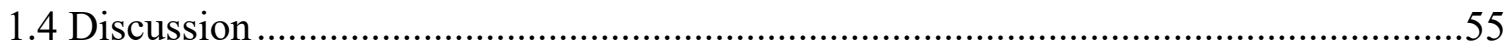

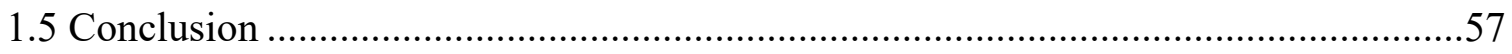

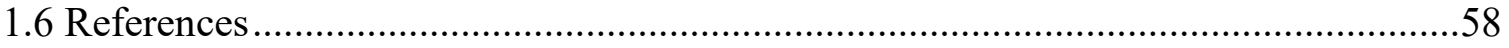




\section{List of figures}

Figure 1 DREAM complex components in mammalian cells. ....................................13

Figure 2 indirect repression of cell cycle genes through p53-p21-DREAM pathway.......17

Figure 3 Models show how soma-to-germline transformation can occur in human cancer cells as a result of dysfunction of both DREAM complex and $\mathrm{Rb}$......

Figure 4 DNA content of the proliferating, serum-starved, and serum released cells of NCI-H1299, SW480, and OVCAR5 cell lines as measured by flow cytometry ...35

Figure 5 DNA content of the proliferating, serum-starved, and serum released cells of MDA-MB-231, SKOV3, and 8MGBA cell lines as measured by flow cytometry

Figure 6 DNA content of the of the proliferating, serum-starved, and serum released cells

T98G cell line as measured by flow cytometry

Figure 7 DNA content of the of the proliferating, serum-starved, and serum released cells of ES-2, OVCAR-3, and OVCAR-10 cell lines as measured by flow cytometry .38

Figure 8 mRNA expression of CT genes in proliferation cells of H1299, SW480, OVCAR-5, SKOV3, 8MGBA, T98G, MDA-MB-231, OVCAR-3, OVCAR-10, and ES-2 cell lines was analyzed by qPCR

Figure 9 mRNA expression of CT genes in proliferation cells of SW480, 8MGBA, T98G, and OVCAR-3 cell lines was analyzed by qPCR

Figure 10 mRNA expression of early cell cycle genes (MCM5, ORC1, and CDC45) in the proliferated cells and the quiescent cells in 8MGBA, H1299, SW480 cell lines was measured by qPCR.

Figure 11 mRNA expression of late cell cycle genes (CCNB2, PBK, and BUB1) in the proliferated cells and the quiescent cells in 8MGBA, H1299, SW480 cell lines was measured by qPCR.

Figure 12 The activity of E2F binding sites (Orc1) and CHR binding sites (Bub1) in the wild type (WT) and the serum-starved cells in the SW480 and H1299 cell lines were analyzed by luciferase reporter assay. 


\section{List of tables}

Table 1.1 Examples of P53 target genes.................................. 18

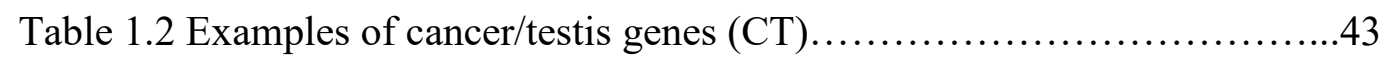

Table 1.3 Examples of DREAM and Rb target genes.........................52 


\section{Acknowledgements}

First, I would like to express my gratitude to God. Next, I thank my father, who deeply encouraged me at the beginning of this journey; he would be so happy. To my mother, my sisters, and my brothers, I am grateful for their continuous support. I want to thank King Abdullah bin Abdulaziz Al Saud for giving me this great opportunity to achieve my master's degree in the United States of America. Also, I thank my country Saudi Arabia, and the Saudi Arabian Cultural Mission (SACM) for support to persevere. I want to thank my dear advisor, Dr. Paul, for his expert advice and encouragement throughout this project. Without his active guidance, I would not have been able to accomplish this endeavor.

I would like to recognize Michigan Technological University MTU, Graduate School, our department, department chair, Dr. Joshi, as well as each committee member Dr. Zhiying Shan and Dr. Ebenzer Tumban, for their efforts. Moreover, finally, I give many thanks to my friends for their support. 


\section{List of abbreviations}

Cancer Testis genes (CT)

The dimerization partner, RB-like, E2F, and multi-vulval class B (DREAM complex)

The retinoblastoma protein $(\mathrm{Rb})$

Cell cycle genes homology region (CHR)

Cell cycle-dependent element (CDE)

CHR-like elements (CLE)

Caenorhabditis elegans (C. elegans)

Clear cell renal cell carcinoma (CCRCC)

Programmed cell death (PCD)

Cyclin-dependent kinase (CDK)

Extracellular matrix (ECM)

Breast cancer cell line (MDA-MB-231)

Colon adenocarcinoma cell line (SW480)

Human ovarian cancer cell line (ES-2)

Human ovarian carcinoma cell line (OVCAR-3)

Human ovarian cancer cell line (OVCAR-5)

Human ovarian cancer cell line (SKOV3)

Human ovarian cancer cell line (OVCAR-10)

Glioblastoma cell line (T98G)

Glioblastoma cell line (8MGBA)

lung cancer cell line (NCI-H1299) 


\section{Abstract}

Misexpression of germline genes like Cancer Testis (CT) genes, called a soma-togermline transformation, is a phenomenon linked to tumorigenesis. However, the mechanisms underlying this phenomenon are poorly understood. A soma-to-germline transformation in Caenorhabditis elegans occurs due to the loss function of the highly conserved DREAM (Dp, Retinoblastoma (Rb)-like, E2F, and MuvB) transcriptional repressor complex. In mammalian cells, the DREAM complex (Muvb core complex, $\mathrm{E} 2 \mathrm{~F} 4 / 5, \mathrm{DP} 1 / 2$, and $\mathrm{p} 130 / \mathrm{p} 107$ proteins), as well as the Retinoblastoma protein (pRb), are implicated in transcriptional repression of cell cycle genes in quiescence or G0. We hypothesize that the expression of CT genes in malignant cells occurs because of the loss of activities of DREAM complex or $\mathrm{pRb}$, similar to how the soma-to-germline transformation occurs in C. elegans. Thus, we expect that cancer cells that express CT genes will either fail to arrest in G0 or display defective repression of key cell cycle genes. To test sensitivity cells to arrest in response to limiting growth conditions, we did flow cytometry to measure the DNA content of 10 cell lines. We found that seven cell lines arrested in $\mathrm{G} 0 / \mathrm{G} 1$, indicating that these cells have downregulated $\mathrm{CT}$ genes expression, and DREAM or $\mathrm{Rb}$ is involved in repressing cell cycle in $\mathrm{G} 0 / \mathrm{G} 1$. This result also suggested that in the cells that did not arrest, both DREAM and $\mathrm{Rb}$ are inactive. Next, to test the mRNA expression of CT genes of all the cell lines, we did mRNA analysis of $\mathrm{CT}$ genes. We found that $\mathrm{CT}$ genes are expressed in proliferating cells of both cells that can arrest under limiting growth conditions and the cells that do not, indicating 
we did not observe a difference in mRNA expression between cells that arrest and cells that do not. To test if $\mathrm{CT}$ misexpression is associated with dysfunction in either $\mathrm{Rb}$ or DREAM, we did further analyses to test early and late cell cycle genes expression in SW480, NCI-H1299, and 8MGBA, comparing the expression of cell cycle cells in proliferating cells to arrested cells in $\mathrm{G} 0 / \mathrm{G} 1$. We found that mRNA expression of early cell cycle genes MCM5, ORC1, and CDC45 are downregulated, as expected since DREAM and Rb regulation of these genes overlap. However, DREAM solely regulates G2/M genes like CCNB2, PBK, and BUB1. We found that G2/M genes were not in significantly downregulated in NCI-H1299 and SW-480 cells, suggesting that DREAM is dysfunctional in these cell lines. As a secondary test of DREAM and Rb function, we performed luciferase reporter assays with promoters of DREAM and $\mathrm{Rb}$ target genes in SW480 and NCI-H1299 cell lines. Surprisingly, we observed that Rb is dysfunctional in SW480 cells. Together, these studies will facilitate future studies into the link between cell cycle regulation and $\mathrm{CT}$ upregulation in cancer cells. 


\subsection{Introduction}

Cancer is a very dynamic complex disease. Over time, it develops from accumulations of genetic and epigenetic alternations as a result of mutations that cause uncontrolled cell division [1]. There are estimated numbers that approximately 1 million new cancer incidences annually in males and more than 900,000 in females between 2010 and 2020 in the U.S. The majority of cancer types expected to increase in males are melanoma, prostate, and kidney cancer. Moreover, breast, lung, and uterine cancer are the most cancer types expected to increase in females [2].

Despite the rapid advance in the fields of biological sciences in an understanding of molecular cancer genetics, how normal cells acquire the malignant characteristics is not completely understood. There are traits that normal cells acquire to transform into cancer are called "Hallmarks of Cancer" [3]. Each hallmark contributes to cancer and can help to understand tumor pathogenesis and the complexity of cancer.

Normal cells have many regulators and pathways that control cell division and prevent DNA damage. Some of these regulators promote cancer development if they are inactivated. For example, one of the critical regulators is the Retinoblastoma $(\mathrm{Rb})$ protein which is inactivated in many human cancerous [4]. The $\mathrm{Rb}$ family proteins including 
$\mathrm{Rb} / \mathrm{p} 105, \mathrm{Rb} 2 / \mathrm{p} 130$, and $\mathrm{p} 107$ control cell cycle progression and DNA replication through interaction with E2F to regulate the gene expression of a set group of genes [5].

Both The retinoblastoma $\mathrm{pRb}$ and DREAM have similar functions in that they induce transcription repression in $\mathrm{G} 0 / \mathrm{G} 1$ by interaction with E2F elements. DREAM has a distinct function from $\mathrm{pRb} / \mathrm{E} 2 \mathrm{~F}$ that it regulates many of cell cycle genes involved in DNA replication, chromosome segregation, DNA repair, histone modification, and chromatin organization including BRCA1, BRCA2, FANCD1, FANCA, CDC6, CDK2, and EZH2 because it can bind to both E2F and CHR sites [6].

The p53-p21-DREAM pathway controls the cell cycle through downregulation the expression of many genes that are involved in cell cycle progression. It regulates more than 250 genes that are associated with cell cycle progression. This pathway targets genes from the G1, G2, and M phase [7]. Thus, it controls all checkpoints, including G1, S, G2, and M. Defects in genes that regulate cell cycle cause aberrant checkpoint control that promotes aneuploidy and chromosomal instability in cancer cells [8].

Previous studies reported that Cancer/testis (CT) genes are ectopically activated in many human cancerous under pathogenesis conditions. CT genes have a pattern of expression that is restricted to normal and tumor cells [9]. Since then, many studies have emerged to address the factors or mechanisms that contribute to re-repression CT genes in human cancer cells. However, the mechanisms that drive the activation of CT genes in 
somatic cells still unknown. Therefore, we propose that the activation of CT genes may be a result of the dysfunction of both transcriptional repressors RB and DREAM in mammalian cells. 


\subsubsection{The six hallmarks of cancer cells}

Cancer cells display unique traits or hallmarks that promote uncontrolled proliferation and malignancy as compared to normal somatic cells. Six cancer hallmarks, in particular, have been defined. These include self-sufficiency in proliferation signals, inactivation of tumor suppressors, enabling of replicative unlimitedly, activation of invasion and metastasis, stimulating angiogenesis, and resistance to cell death [3]. Alterations of many functionally overlapping molecular pathways govern the prevalence of each cancer hallmark in a given tumor type. There is a need to identify the potential causes that drive these alternations in all types of cancers. Therefore, research into the molecular pathways that contribute to these six cancer hallmarks continues to drive the development of new methods to combat cancer.

Regarding the first hallmark, genetic or epigenetic alternations in tumors enable them to require the ability to sustain signals that promote chronic division and proliferation [3] [10]. Many of genetic and epigenetic changes are oncogenic mutations that affect signaling pathways, leading in turn to an anti-apoptotic potential, deregulated mitogenic, and increased motility in tumor cells [11]. For instance, increased pRb phosphorylation leads to loss of Rb function, and it occurs in most cancer types [12]. Oncogenic mutations cause upregulation of transcription of cyclin-dependent kinase (CDK) that mediate phosphorylation of $\mathrm{pRb}$ during the transition from $\mathrm{G} 1$ to $\mathrm{S}$ phase. It occurs in response to proliferative signals, that causes it to release of E2F, which promotes entry into $\mathrm{S}$ phase [13]. Inactivation of the tumor suppressor signaling 
pathways leads to remove negative regulators of signaling that play an essential role in proliferation and progression in cancer cells [10].

Regarding the second hallmark, $\mathrm{pRb}$ and $\mathrm{p} 53$ functions are inactivated in most tumor cells and are a typical example of cancer cells evading tumor suppressor pathways [3]. These proteins have the role of suppressing and controlling cell growth and inducing senescence (cellular aging) and programmed cell death (PCD) [14]. The p53 gene regulate many functions of the cell cycle, including repairing damaged DNA, arresting growth, and apoptosis [15]. Gain- or loss-of-function in $\mathrm{pRb}$ or $\mathrm{p} 53$, which regulates proliferation, can trigger senescence, a normal process involving the irreversible arrest of normal cells [16] [17]. For instance, loss of function in p53 causes defects in cell cycle checkpoints and DNA damage repair, leading to chromosomal instability and increased mutation rates [18]. The majority of human cancer have mutated $\mathrm{pRb}$ or $\mathrm{p} 53$ genes because of the loss of functions of $\mathrm{p} 53$ and $\mathrm{pRb}$ pathways that promote tumorigenesis and tumor progression [19].

The third hallmark of tumor cells is their ability to resist cell death, or apoptosis [3]. Apoptosis is a tightly controlled mechanism involving energy-dependent cascades [20]. Apoptosis has two pathways: the intrinsic or mitochondrial pathway and the extrinsic pathway. The intrinsic signaling pathway involves intracellular signals that are regulated by the mitochondria [20]. In the latter case, the cell receives cell-death signals from outside itself, which induce cellular suicide [21]. The evading of apoptosis in cancer cells can occur by different mechanisms, such as the loss of function of the p53, 
overexpression of survival signals for example Igf1/2 during the cell death pathway, and overexpression of antiapoptotic $\mathrm{Bcl}-2$ proteins that regulate apoptosis by binding to proapoptotic triggering proteins such as Bak and Bax, thereby suppressing their functions [22]. In cancer cells, there is an imbalance between cell death and cell proliferation, and the cells that should undergo apoptosis did not receive apoptotic signals. Therefore, understanding apoptosis is essential because it can give us insight into the pathogenesis and design an effective treatment of cancer.

The fourth hallmark of cancer cells is their capacity for replicative immortality [3]. The telomere, which is made by telomerase enzyme, is cap-like nucleoproteins found at the tips of chromosomes to protect them from fusion and stabilizes the chromosome and the DNA replication process in the eukaryotic cells [23] [24].

Normal human somatic cells pass through multiple cell divisions, then enter senescence and then eventually apoptosis. During multiple replication cycles, the telomeres shorten, and telomerase activity becomes repressed. However, in cancer cells, telomerase typically activated during immortalization [25]. Due to either genetic or epigenetic alterations, in malignant tumors, the telomerase lengthens; the tumor cells thus show high levels of telomerase activity during the process of immortalization, leading to chromosomal instability [26]. Telomere length besides telomerase activity play a crtical role in oncogenesis. Thus, it is important to focus on understanding the mechanisms that trigger the reactivation of telomerase in cancer cells, because it can help to discover promising biomarkers that can contribute to detect cancer at early stages [27]. 
The fifth hallmark is the initiation of tumor angiogenesis processes, which is the secretion of angiogenic growth factor proteins by cancerous tumors that stimulate to form new blood and lymphatic vessels [3]. Cancer cells form new vascular network because of proliferation, and metastatic spread requires access to oxygen, nutrition, and removal of the waste products [28]. It allows cancer cells to leave the primary site and migrate through the bloodstream to invade secondary sites to form colonies, as well as to metastasize in the late stages of the disease [3]. Previous histological studies on human tumors have considered a link between p53 and angiogenesis. Tumors with mutated p53 are significantly more vascularized and aggressive than those that have retained functional p53 [29]. The angiogenesis process is essential for cancer cells because it enables them to form vascular networks through the bloodstream from the primary site and migrate to settle a secondary site or metastasis site. Therefore, prevention of tumor angiogenesis processes can combat neoplastic growth at the primary site and prevent metastasis of tumor in late-stage, that will allow for treatment by therapeutics.

The sixth hallmark is that tumor cells can penetrate surrounding tissues and metastasis. Metastasis is observed when malignant tumors migrate from the primary site by the bloodstream or lymph vessels, to form new tumors at other locations [3]. The tumor cells interact both cell-to-cell and within the extracellular matrix (ECM). The ECM contributes to changes in cell adhesion, thus providing a conducive environment in which the tumor can grow. The $\mathrm{pRb}$ protein can mediate these pathways and contribute to dissociating tumor cells from their original location [12]. Metastasis may occur due to defects in the epidermal growth factor receptor (EGFR), which regulates the cellular and 
homeostatic proliferation of epithelial tissues in normal cells [30]. Analysis of EGFR expression levels in vitro shows that metastatic cells are highly expressive of EGFR when compared with non-metastatic cells in human colon cancer (HCC) [31]. Besides the focus on developments of methods to detect the tumor at an early stage. It is crucial to understand the mechanisms underlying the metastasis because once tumors spread and migrate from the original site, they can be incurable and fatal [32].

The six cancer hallmarks that cancer cells are acquired to develop tumors are important to understand the pathogenetic mechanisms of a very complex disease such as cancer, and to support therapeutic strategies to fight this disease. They are including stimulating proliferative signaling, inactivating growth suppressors, enabling replicative unlimitedly, resisting apoptosis, inducing angiogenesis, enabling invasion, and metastasis [3].

pRb and p53 are significant players in cancer development. However, many questions remain as to how $\mathrm{pRb}$ and $\mathrm{p} 53$ regulate cell cycle genes, and how $\mathrm{Rb}$ and DREAM overlap different in their functions to repress cell cycle genes in G0, G1, S, G2 and $\mathrm{M}$ phase.

Since it is known that p53 acts as repressor and activator, how its functions can influence the p53-DREAM pathway is still not completely understood. Besides, the mechanism of repression cell cycle genes indirectly by the p35-DREAM pathway 
remains unsolved issues, and it is a big challenge for scientists because it showed unexpected findings that are raising new questions. 


\subsubsection{Dysregulation of $\mathrm{pRb}$ is associated with tumor development}

$\mathrm{pRb}$ is the first known tumor suppressor that regulates cell cycle progression [50]. The Rb family consists of three homologs, pRb (RB1), p107 (RBL1), and p130 (RBL2), also known as pocket proteins [51]. The retinoblastoma protein inhibits gene transcription by activating the E2F-DP transcription factor at E2F sites to repress gene expression of genes required in transition from $\mathrm{G} 1$ to $\mathrm{S}$ phase leading to cell cycle arrest at early $\mathrm{G} 1$ phase [52]. Genetic studies of knockout Rb genes show that they have many functions and participate in multiple cellular processes such as gene expression and regulation, cancer development, and cell cycle control [53]. The retinoblastoma tumor suppressor gene $(\mathrm{RB})$ is inactivated in the most of human cancers due to direct mutation or deletion, such as small-cell lung carcinoma, and retinoblastoma, or indirectly by alterations of the activities of the upstream regulators [54].

In tumor cells containing oncogenic mutations, the $\mathrm{Rb}$ pathway is disruption. Following mitogenic stimulation, P21/CDKN1A inactivate cell cycle by inhibiting cyclin-dependent kinases (CDKs) function that cause phosphorylate of the pocket proteins $\mathrm{p} 107 / \mathrm{p} 130$ [55]. Phosphorylation of Rb proteins leads to release of E2F from inhibition and activate of the cell cycle genes that are required for S phase entry [52].

The $\mathrm{pRb}$ regulates cell division in the nucleus by preventing the progression of the cell cycle. In normal cells, $\mathrm{Rb}$ is found in non-phosphorylated status, and bound to 
E2Fs in G0/G1 [52]. During the phosphorylation of Rb that occurs by cyclin D-CDK4 and cyclin D-CDK6, it prevents Rb association with E2Fs. Thereby, it facilitates E2Fs to activate the transcription of genes that are essential for S phase entry [56]. Altered expression of cell cycle regulators, including cyclin $\mathrm{D}, \mathrm{CDK} 4$, and their principal inhibitor $\mathrm{p} 16$, is very common in cancer cells. By the end of G1, binding cyclin proteins cause inactivation by cyclin cd complex. This inactivation causes $\mathrm{pRb}$ to release bound E2F transcription factors, which promote the expression of genes that are essential for cell progression from $\mathrm{G} 1$ to $\mathrm{S}$ phase [56]. Loss of functions of $\mathrm{Rb}$ is common in the majority of human cancers by different mechanisms. For instance, inactivation of $\mathrm{Rb}$ in human papillomavirus (HPV) is a result of E7 which is oncogenic protein that binds to $\mathrm{Rb}$ and inhibits its activity from repress damaged cells leading to cell proliferation [57].

Overlapping activations of $\mathrm{pRb} / \mathrm{E} 2 \mathrm{~F}$ and DREAM are required for cells to enter G0 or quiescence through indirectly repression by the p53-DREAM pathway. The $\mathrm{pRb} / \mathrm{E} 2 \mathrm{~F}$ and DREAM complexes promote cell cycle arrest via CHR or E2F sites in $\mathrm{G} 0 / \mathrm{G} 1$ phases. $\mathrm{Rb}$ and its related proteins $\mathrm{p} 130$ and $\mathrm{p} 107$ have a master function to repress gene transcription by binding to E2F sites and inhibit their activities during G1 to S phase transition [7].

The pRb-related proteins p130/p107 become hypophosphorylated as a result of the expression of $\mathrm{p} 21 / \mathrm{CDKN} 1 \mathrm{~A}$ that leads to gathering proteins that are involved in formation DREAM complex for repressing transcription through E2F or CHR promoter 
sites. The p130/p107, E2F4, and E2F5 are bound via E2F cities during transcriptional repression of the cell cycle [50].

The p130/p107 are essential components of the DREAM complex that mediate gene repression [7]. Because of that, knockdown pocket proteins p107/p130 lead to a loss of DREAM function as a transcriptional repressor [58]. However, how pRB/E2F and DREAM complexes overlap functions to coordinate cell cycle checkpoints remains unclear. 


\subsubsection{DREAM complex pathway in repression cell cycle genes}

DREAM complex is a highly conversed protein that represses cell cycle genes during quiescence in mammalian cells [6]. It is composed of p107 or p130, E2F4-5/DP and MuvB core (figure 1) [58] [68]. It plays a critical role in the downregulation of cell cycle gene expression through quiescence and early G1 [69]. It binds to DNA promoters via the cell cycle genes homology region (CHR) or E2F sites through the cell cycle. It also binds CLE (CHR like element) and CDE (cell cycle-dependent element) sites on DNA. However, it cannot bind to DNA sites by CDE or CLE alone; it requires support by either CHR or E2F [70].

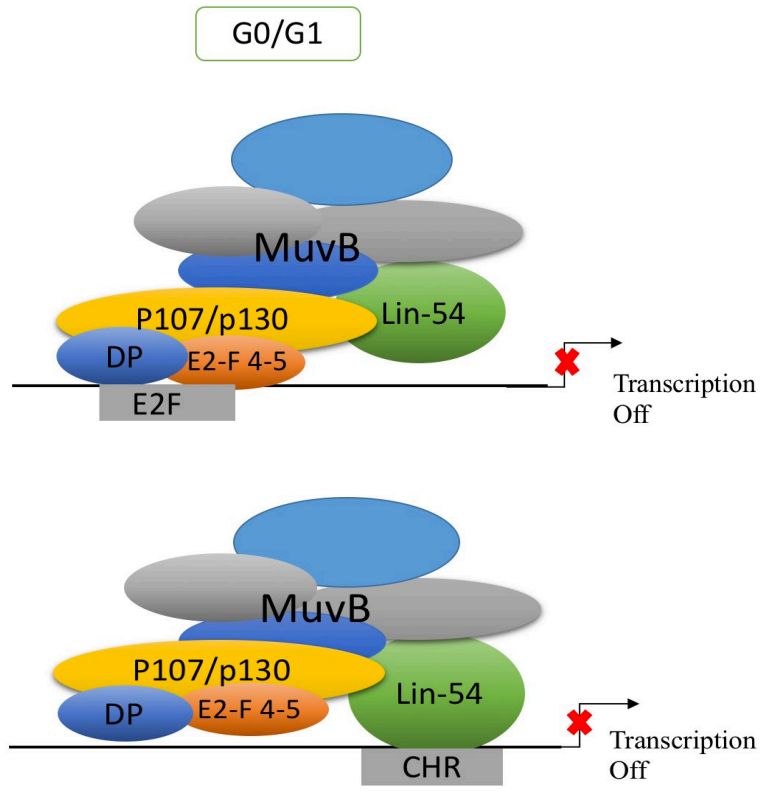

Figure 1 DREAM complex components in mammalian cells. DREAM binds $\mathrm{E} 2 \mathrm{~F} / \mathrm{CHR}$ or E2F/CLE, and CHR/CDE sites during G0/G1 to repress the transcription of the cell cycle genes in G0 and early G1 based on [7]. 
Cell cycle genes have a repression mechanism indirectly through the p53-p21DREAM pathway. In G0/G1, cell cycle genes are repressed by the interaction of DREAM complex and RB-E2F through E2F sites on DNA [71]. In respond to DNA damage, $\mathrm{p} 53$ becomes activated, which leads to induce the expression of p21/CDKN1A. It produces $\mathrm{p} 21$ that form $\mathrm{p} 21 / \mathrm{CDKN} 1 \mathrm{~A}$ that interacts with cyclin-dependent kinases (CDKs) and inhibits activity. It leads to phosphorylate the pRB-related proteins p107, and p130. As a result of that, $\mathrm{p} 107$ and p130 become hypophosphorylated and can form DREAM complex with other proteins to repress the transcription by binding DREAM to E2F or CHR sites [7]. In late G1, and early S phase, DREAM complex dissociates from repressive components $\mathrm{E} 2 \mathrm{~F} / \mathrm{pRB}$ and $\mathrm{p} 107$ and $\mathrm{p} 130$ and binds to transcriptional activators B-MYB to form B-MYB-MuvB complex that switch the repression to activation.

In late S and early G2 phase, FoxM1 (Forkhead box M1) binds to the complex and form FOXM1-MMB complex. The B-MYB and FoxM1 coordinate gene expression of many cell cycle genes during $\mathrm{S}$ and G2/M phases [72]. The MuvB core comprises of LIN9, LIN37, LIN52, RBBP4 proteins, and LIN54, in contrast to the DREAM complex, it does not have the E2F, Rb, and DP proteins and only binds to CHR promoter sites [7]. MuvB interacts with BMYB and FOXM1 to regulate gene expression through S phase and G2/M [6]. Because MuvB based complex can change its formation, it binds to BMYB or FoxM1 and forms B-MYB-MuvB (MMB) that turns repression to activation when LIN54 binds to CHR site with peaks expression in G2/M phases [7]. 
p53-DREAM pathway is a critical pathway because it contributes to cell cycle arrest, and it represents a promising therapeutic target for cancer. Disruption of the p53DREAM pathway results in loss of checkpoint control. Importantly, many of the p35DREAM pathway genes are involved in many vital cellular processes such as chromosomal segregation. Hence, the deregulation of their expression contributes to aneuploidy and chromosomal instability in cancer cells. 


\subsubsection{Downregulation expression of cell cycle genes by p53}

p53 is a critical tumor suppressor that downregulates many cell cycle genes leading to cell cycle arrest or apoptosis [15] [59]. It is a transcriptional activator and repressor, as well [60]. The p53 gene is mutated in more than $50 \%$ of human tumors [61]. Many target genes of p53 discovered that mediate its function as a tumor suppressor (Table 1.1) [69].

Mutations in p53 are oncogenic and contribute to neoplastic transformation [62] [63]. The activation of p53 is regulated by mouse double minute 2 homolog (MDM2). MDM2 is an important negative regulator of p53 that inhibits its activity, and it is overexpressed in sarcoma. Upon activation, the MDM2 gene interacts with p53 by directly binding to its transactivation domain and inhibits its transcriptional activity leading to ubiquitination degradation of p53 [64]. It downregulates many cell cycle genes from G1 through cytokinesis. It responds to stress signals in many ways by regulation cell cycle pathway, DNA repair, apoptosis, and senescence [65]. It stimulates many target genes that promote apoptosis, including, but not limited to, PIG3 (tumor protein p53 inducible protein 3), Bax (BCL2-associated X protein), and PUMA (p53 upregulated modulator of apoptosis) [66].

The p53 gene has an indirect repression mechanism, which is regulated by the expression of some downstream effectors such as p21, CDN1A, miRNAs, and E2F7 [67]. Upon activation, p53 downregulates many cell cycle genes through the p53-DREAM 
pathway (figure 2). DREAM and RB-E2F have overlapping functions, and they promote transcriptional repression and cell cycle arrest by p53. Activation of p53 leads to cell cycle arrest during G1 and G2/M checkpoints. It recruits the DREAM complex to downregulate the expression of a set range of essential of the cell cycle progression [7]. In response to DNA damage, p53 activates p21 that inhibits cell division-stimulating protein (cdk2) activity leading to indirect transcriptional repression and stop cell cycle progression and ultimately arrests the cell in G0/G1 [7].

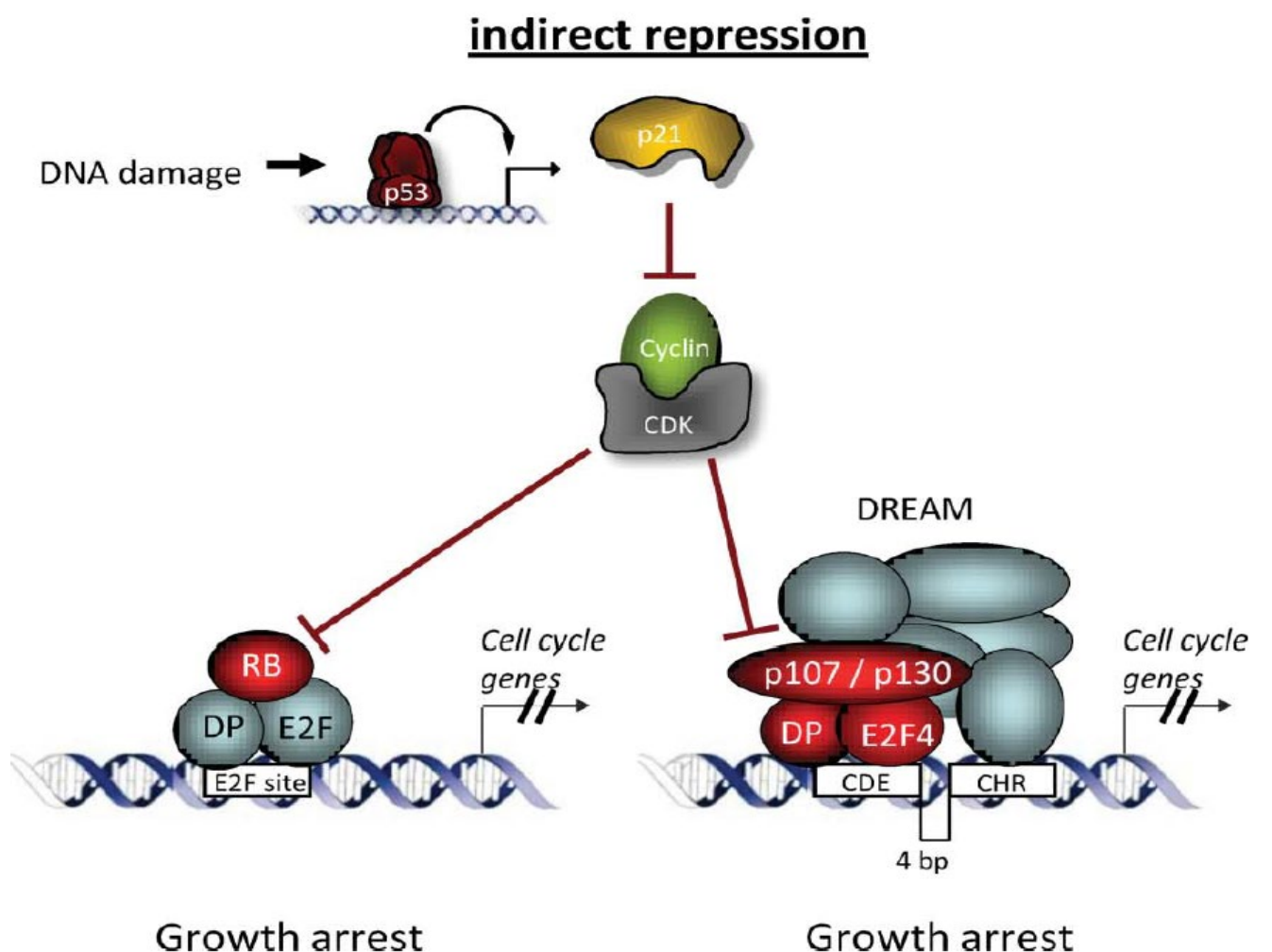

Figure 2 indirect repression of the expression of the cell cycle genes through p53p21-DREAM pathway. p53 activates p21 in response to DNA damage, which stops the cell cycle by inhibiting Cyclin CDK complexes based on [70]. 


\begin{tabular}{|c|c|c|c|c|c|c|c|c|c|}
\hline BLOC1S2 & SESN1 & FBXO22 & RPS27L & TNFRSF10B & DDB2 & RRM2B & DCP1B & FAS & GADD45A \\
\hline ACTA2 & TRIAP1 & PLK2 & BAX & AEN & EDA2R & BTG2 & TP53INP1 & ZMAT3 & PLXNB2 \\
\hline ASCC3 & POLH & XPC & GDF15 & FAM198B & FBXW7 & TRAF4 & SLC30A1 & ZNF219 & PIDD1 \\
\hline EPS8L2 & TM7SF3 & TMEM68 & DRAM1 & ANKRA2 & SUSD6 & PRKAB1 & ORAI3 & ZNF79 & NINJ1 \\
\hline E2F7 & REV3L & S100A2 & TYMSOS & $\mathrm{FCHO} 2$ & TRIM32 & PMAIP1 & TSKU & SPATA18 & AMZ2 \\
\hline SRA1 & ISCU & GRHL3 & PANK1 & TP53I3 & CCDC90B & PRKAB2 & RNF19B & FAM210B & SERPINB5 \\
\hline MAST4 & MCC & FDXR & COL7A1 & CDIP1 & FAM212B & IGDCC4 & RETSAT & EPHA2 & RETSAT \\
\hline PSTPIP2 & CPSF4 & TSPAN11 & CSNK1G1 & ASTN2 & TRIM35 & KCTD1 & IRF2BPL & SEMA3B & SCRIB \\
\hline DGKA & WDR63 & ANXA4 & ATF3 & CD82 & HHAT & USP15 & CD82 & FLRT2 & BHLHE40 \\
\hline CYFIP2 & BLCAP & BTG1 & BLCAP & SYTL1 & KRT15 & MICALL1 & RRAD & FAM84B & SLC9A1 \\
\hline FAM84B & AKAP9 & BCL6 & ADIRF & PPFIBP1 & TXNIP & ADIRF & ITGA3 & PPFIBP1 & TXNIP \\
\hline HES1 & HES1 & DGKA & PSTPIP2 & CMBL & WDR63 & KRT15 & ANXA4 & FLRT2 & IER5 \\
\hline
\end{tabular}

Table 1.1 Examples of P53 target genes 


\subsubsection{Aberrant expression of cancer/testis (CT) genes in somatic cells}

The processes that drive the evolution of germ cells and tumor cells share similar biological characteristics. Recognition of the biological features of the trophoblast cells tumor cells led to propose a hypothesis called the 'trophoblastic theory of cancer', which suggests that cancer cells emerge from germ cells that unable to continue migrating to the gonads during embryonic development [34]. Trophoblast cells are known as the cells that form the outside layer of the blastocyst to become the placenta in mammalian cells [35]. Since then, many genes have been discovered that are expressed in germ cells, trophoblasts, and tumors, and these are known as cancer/testis (CT) genes [9]. CT genes encode immunogenic antigens called Cancer Testis Antigens (CTA). These antigens can evoke cellular and humoral immune responses in patients with cancer [36]. Because of the discovery of CT gene expression in many cancer cell types, one of the theories proposed that the 'misexpression' of germline genes in cancer cells is as a result of a silenced gametogenic program, it being abnormally activated in somatic cells. Thus, the activation of gametogenic program is one of the factors that drive tumorigenesis [37].

It is during early embryonic development that the fate of all cells is determined.

The cells become either non-reproductive somatic cells or germ cells that eventually form the germline that produces an organisms' gametes, sperm, or eggs [38]. Germline genes are normally expressed in the male testis or female ovaries and placenta [39]. Somatic cells silence or turn off germline genes, but cancer cells often misexpress a class of 
germline genes called CT genes. The expression of the germline in malignant cells is called the soma-to-germline transformation, a de-differentiation phenomenon observed in somatic cells in model organisms like Drosophila melanogaster and Caenorhabditis elegans [40]. Misexpression of germline genes appears to be linked to cancer initiation and progression in mammalian somatic cells. Aberrant expression of CT genes causes chromosome missegregation and aneuploidy in cancer cells [36]. Loss of CT genes repression in tumor cells is a hallmark of cancer disease. Thus, understanding genetic and epigenetic alterations in tumor cells that occur as a result of misexpression of $\mathrm{CT}$ is important, because it can help to understand the mechanisms that contribute to inhibit expression of the $\mathrm{CT}$ genes in somatic cells to protect their fates during development [40].

CT genes are ectopically expressed in many types of cancer cells, and classified into two groups, depending on chromosome localization [36]. The first group is CT genes encoded by X chromosome genes; $10 \%$ of these genes are expressed in tumor cells and contribute to tumor evolution [41]. The second group comprises CT genes that are encoded by non-X chromosome genes and found on autosomal chromosomes expressed during meiosis [42]. The molecular and physical functions of most CT genes remain unclear [36]. Because CT genes are ectopically expressed in many human cancer types, it is necessary to understand the mechanisms that drive their activation in tumors. CT genes are frequently expressed in tumor types such as bladder cancer, lung carcinomas, hepatocellular carcinomas, melanomas, and ovarian cancer [43]. Although some cancer types show poorly expressed CT genes, such as glioblastoma and clear cell 
renal cell carcinoma (CCRCC), melanomas, and lung carcinomas show abundant overexpression of CT genes [44]. The melanoma antigen gene (MAGE) family is frequently overexpressed in lung adenocarcinomas, while semenogelin 1 (SEMG1) is frequently overexpressed in colon cancer. Basal cell breast cancer shows enriched expression of melanoma-associated antigen 3 (MAGEA3), actin-like 8 (ACTL8), and chromosome X open reading frame 6 (CXorf6). Magnoid lung adenocarcinomas show overexpressed MAGEA1, MAGEA3, MAGEA4, MAGEA12, and CSAG1[44]. Currently, the mechanism underlying how each CT gene is misexpressed in such a wide variety of cancer cell types remains unknown.

CT proteins evoke an immune response in cancer cells and have an expression pattern that is specifically associated with tumor cells and not found in most normal tissues. This makes them unique targets for immunotherapy in cancer treatment [45]. Despite the fact that understanding the expression pattern of these genes would help to identify driver genes in cancer, few studies have investigated why and how the oncogenic mechanisms of CT gene expression impact human somatic tissues.

Research on CT genes presents a challenge for scientists. Firstly, most studies that have analyzed the expression of CT genes have involved just a small number of samples [46]. Secondly, many CT genes remain uninvestigated in terms of how they abnormally reactivated and whether their expression can trigger, or is associated with, tumor progression. Although the mechanisms that drive CT co-expression in somatic and cancer cells remain poorly understood, ectopic expression of CT genes is known to reflect a 'cell 
identity crisis. Normal cells are able to change their identity under pathological conditions. Most cancer cells arise from the epithelial cells that form the skin. In breast cancer, for instance, cells change their epithelial identity and acquire invasive and metastatic characteristics - a process which is known as epithelial-mesenchymal transition (EMT) [47].

In the C. elegans model system, a soma-to-germline transformation occurs as a result of loss-of-function mutations in a homolog of the tumor-suppressor $\mathrm{pRb}$, called lin35. The loss-of-function of lin-35 leads to misexpression of the germline-specific gene pgl-1 [48]. Similarly, the mechanism that drives soma-to-germline transformation in C. elegans might also contribute to the reactivation of CT genes in mammalian somatic cells, though a variety of factors may influence this transformation in human somatic cells [49]. Thus, this study hypothesizes that ectopic expression of CT genes in human somatic tissues is associated with dysfunction of both the transcriptional repressors DREAM and RB-E2F complexes by oncoproteins. 


\subsubsection{Study hypothesis}

In our project, we are addressing the factors that drive the soma-to-germline transformation that occurs in cancer cells in mammalians. In particular, we focus on how the loss of both transcriptional suppressors can promote ectopic expression of Cancer/testis genes (CT) in human cancers. Our hypothesis suggests that cancer cell lines that have $\mathrm{Rb}$ and DREAM complex that are functioning normally or that have either DREAM or $\mathrm{Rb}$ is dysfunction can exit cell cycle under limiting condition (figure 3). However, cancer cells that are lacking both DREAM complex and Rb can not exit cell cycle therefore they can proliferate normally under limiting growth conditions (figure 1A and1B). Thus, we propose that misexpression of CT genes in tumor cells is associated with loss of functions of both DREAM and Rb.

First, we used Flow cytometry to measure the DNA content of 10 cell lines. We found that the majority of the cell lines, a total of seven of the cell lines arrested in G0/G1, under limiting growth conditions. A total of three of the cell lines did not arrest in G0/G1. Next, we analyzed the mRNA expression of many CT genes in proliferating cells of all the cell lines. However, we did not observe specific gene expression pattern between all the cell lines. Then, we analyzed the mRNA expression of Rb and DREAM G0/G1 and G2/M target genes in 8MGBA, NCI-H1299, SW480 cell lines to test if the misexpression of the $\mathrm{CT}$ genes is associated with loss of functions of DREAM or Rb. The mRNA expression results suggest that DREAM and $\mathrm{Rb}$ G0/G1 target genes are still down-regulated in serum-starved cells as compared to proliferating cells, however low 
fold-change levels might indicate loss of function of either DREAM or Rb. Analysis of G2/M late cell gene expression in serum starved cells suggest that DREAM is dysfunctional in SW480 and H1299 cell lines. However, luciferase reporter analyses revealed that $\mathrm{Rb}$ is dysfunctional in SW480 cells. Together, these studies will facilitate future studies into the link between cell cycle regulation and CT upregulation in cancer cells.
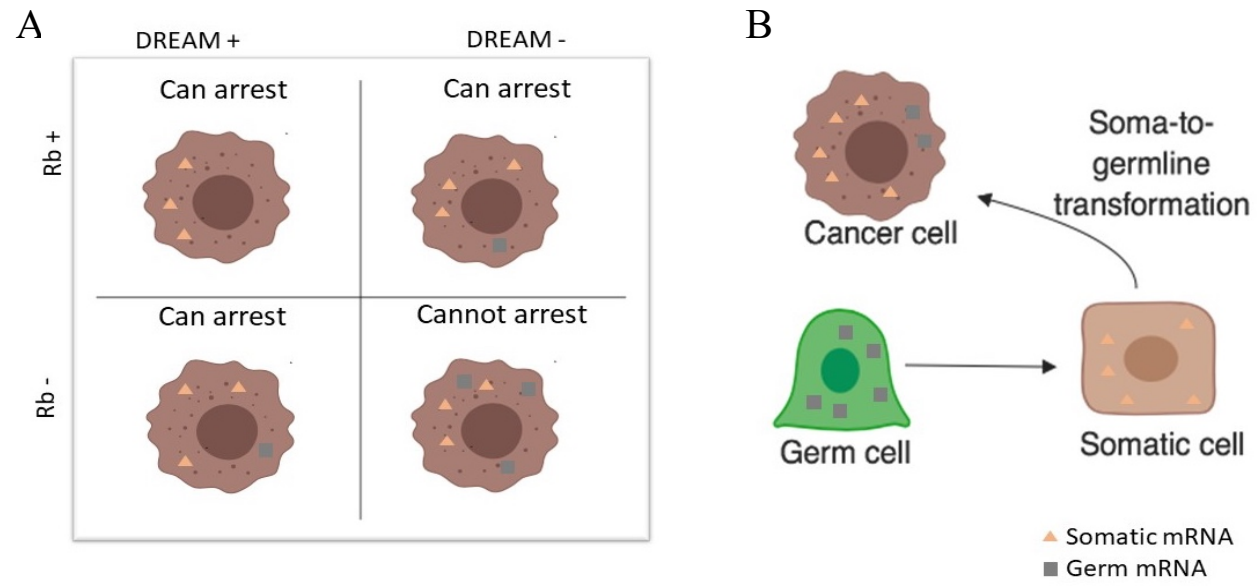

Figure 3 Models show how soma-to-germline transformation can occur in human cancer cells as a result of dysfunction of both DREAM complex and $\mathbf{R b}$.

(A) A model of DREAM and Rb activity in human cancer cells, both DREAM and $\mathrm{Rb}$ arrest cell cycle in $\mathrm{G} 0 / \mathrm{G} 1$, and they can arrest cell cycle even if one of them is dysfunction, however if they both are dysfunction they cannot arrest cell cycle expression in response to limiting growth condition.

(B) A model describes how soma-to-germline genes transformation can occur in human cancer cells. 


\subsection{Methods}

\subsubsection{Cell culture}

The human ovarian carcinoma OVCAR3 cells, SKOV3 human ovarian cancer cells, ES-2 ovarian cells, lung cancer cell line NCI-H1299, human ovarian cancer cells OVCAR5, and human ovarian cancer cells OVCAR10 were cultured in RPMI medium supplemented with $10 \%$ fetal bovine serum (FBS). The SW480, MDA-MB-231, T98G, and 8MGBA cells were cultured in DMEM medium with $1 \%$ Glutamax and $10 \%$ FBS. The cells were plated in 6-well plates (250,000 cells) for each well. The cells were separated into three groups; proliferated cells were cultured for 24 hours to reach $80-90 \%$ confluency. Serum starvation added to the cells for 48 hours. After serum starvation, serum release added to the cells for 24 hours. Cells were washed once with PBS before added serum starvation or serum release. At $24,48 \mathrm{~h}$, adherent cells were detached using trypsin. The cells were starved by $2 \mathrm{~mL}$ of serum starvation containing RPMI medium supplemented with $1 \%$ penicillin/streptomycin or DMEM with $1 \%$ penicillin/streptomycin and 1\% Glutamax for 48 hours, and re-stimulated by added $2 \mathrm{~mL}$ of serum released containing either DMEM or RPMI medium with $20 \%$ fetal bovine serum (FBS), 1\% Glutamax and 1\% penicillin/streptomycin for 24 hours and seeded at different times. Proliferating cells were seeded after 24 hours, serum-starved cells after 48 hours, and serum released after 24 hours. All the cell lines were maintained at $37^{\circ} \mathrm{C}$ and $5 \% \mathrm{CO} 2$. 


\subsubsection{Flow Cytometry}

To measure DNA content, all cell lines were plated in 6 well-plates $(125,000$ cells per well). They were grown in DMEM or RPMI medium for 24 hours. The cells were grown under serum starvation for 48 hours, and serum released for 24 hours after the serum starvation. The cells were trypsinized and stored in $500 \mu \mathrm{l}$ of $70 \%$ Ethanol at -20 ${ }^{\circ} \mathrm{C}$ overnight. Then, they were centrifuged at $4,000 \mathrm{rpm}$ for 2 minutes at $4{ }^{\circ} \mathrm{C}$. Next, they were resuspended in PBS that contains $0.25 \%$ Triton X-100. They were incubated on ice for 15 minutes and spun for 2 minutes at $4,000 \mathrm{rpm}$ at $4{ }^{\circ} \mathrm{C}$. They were resuspended in $500 \mu \mathrm{l}$ PBS, $0.5 \mu \mathrm{l}$ of $10 \mu \mathrm{g} / \mathrm{mL}$ Rnase $\mathrm{A}$, and $10 \mu \mathrm{l}$ of $1 \mathrm{mg} / \mathrm{mL}$ Propidium Iodide (PI). Finally, they were transferred to the FACS tubes and stored for 30 minutes at room temperature before analyzed by Flow cytometry. 


\subsubsection{RNA extraction}

Isolation of total RNA with $500 \mathrm{~mL}$ of TRIZOL reagent and incubated overnight. Chloroform was added and transferred to a phase-lock tube and mixed. Then, it was incubated at room temperature for 10 minutes. The cells were centrifuged at $4{ }^{\circ} \mathrm{C}$ for 15 minutes at 12,000 RCF. Next, the top aqueous layer was transferred to RNase free tube. Isopropanol was added and mixed and incubated at room temperature for 5 minutes. Then, the tubes were centrifuged at $4{ }^{\circ} \mathrm{C}$. for 8 minutes at $12,000 \mathrm{RCF}$. The liquid was removed, and Ethanol/Nuclease-free water was added and centrifuged for 5 minutes at $12,000 \mathrm{RCF}$. The liquid was removed from the tubes and centrifuged for 1 minute at $12,000 \mathrm{RCF}$. The liquid was removed, and the tubes left open to dry by air for less than 4 minutes. Lastly, Nuclease-free water was added and stored at $-70{ }^{\circ} \mathrm{C}$ overnight. 


\subsubsection{Reverse Transcription}

The concentration of RNA was between 200-500ng, and RNase-free water total $8 \mu \mathrm{L}$. We made the first DNase 1 treatment master mix a total of $2 \mu \mathrm{L}$ per sample. The first master mix for RNA 500ng concentration was $1 \mu \mathrm{L}$ of $10 x$ DNase buffer, $0.25 \mu \mathrm{L}$ of DNase I, and $0.75 \mu \mathrm{L}$ of RNase-free water per sample. The mix was added to each sample $(2 \mu \mathrm{L}$ per sample). The tubes were incubated for 15 minutes at room temperature. Next, mM EDTA was added to the tubes $\left(1 \mu \mathrm{L}\right.$ per sample). The tubes incubated for 10 minutes at $65^{\circ} \mathrm{C}$. The tubes were then transferred to an ice bath. We prepared RT-PCR master mix that contains of 10x RT buffer $(0.2 \mu \mathrm{L}), 25 x d N T P$ Mix $(100 \mathrm{mM})(0.8 \mu \mathrm{L}), 10 x$ RT Random Primers $(0.2 \mu \mathrm{L})$, MultiScribe RTase $(1 \mu \mathrm{L})$, and Nuclease-free water $(3.2 \mu \mathrm{L})$ the total volume reaction was $9 \mu \mathrm{L}$ per sample. A total of $9 \mu \mathrm{L}$ of the mix was added to each sample and mixed and centrifuged. The samples ran on ABI simpliAmp cycler. The samples were diluted $1: 2.5$ or 1:5 per 500 ng RNA amount and the ddH2O was $30 \mu \mathrm{L}$ or 8 $\mu \mathrm{L}$ after the dilution they were transferred to a fresh $1.5 \mathrm{~mL}$ tube. 


\subsubsection{Quantitative PCR}

We made a dilution with ddH2O for each Cancer/Testis gene (CT) that we used before making the primers. Next, we made a master mix reaction that contains $5 \mu \mathrm{L}$ of $2 \mathrm{x}$ SYBR Green (ABI), $0.5 \mu \mathrm{L}$ of $10 \mu \mathrm{M}$ Forward primer, $05 \mu \mathrm{L}$ of $10 \mu \mathrm{M}$ Reverse primer, $2.0 \mu \mathrm{L}$ of ddH2O per sample. The total volume was $8 \mu \mathrm{L}$ per well. The plate was centrifuged at $1500 \mathrm{rpm}$ for 2 minutes and added to the qPCR machine and ran on ABI QuantStudio 3. 


\subsubsection{Transfection and luciferase assays}

To test promoter activities of DREAM with luciferase reporter assays, H1299, SW480 cell lines were cultured in RPMI medium supplemented with $10 \%$ fetal bovine serum (FBS) or DMEM medium with 1\% Glutamax and 10\% FBS. SW480-NCI-H1299 cells were plated in 24 well-plates ( 25000 cells per well) and incubate for 24 hours. Each well was washed with $0.5 \mathrm{~mL}$ PBS. The medium was replaced with serum-free media containing RPMI medium supplemented with $1 \%$ penicillin/streptomycin or DMEM with $1 \%$ penicillin/streptomycin and $1 \%$ Glutamax for 24 hours followed by transfected.

For transfection, Lipofectamine 3000 reagent mixes were made that contain: first mix 25 $\mu \mathrm{L}$ of serum-free media, 50ng pGL4.70 Renilla, and 200ng pGL4.10 reporter, and 0.5 of $\mu \mathrm{L}$ P3000 reagent per well. The second mix was $25 \mu \mathrm{L}$ of serum-free media, and $1 \mu \mathrm{L}$ of Lipofectamine 3000 regent. The second mix was added to all the tubes of the first mix. The tubes were incubated at room temperature for 15 minutes. Next, $50 \mu \mathrm{L}$ of the mix was added to each well and incubated for 48 hours. The reporters are Bub1 (WT), Bub1 (CHR), ORC1(WT), and ORC1(E2F). After 48 hours, the wells were washed with 0.5 $\mathrm{mL}$ PBS, and then $100 \mu \mathrm{L}$ 1x Passive lysis buffer (PLB) was added to each well. The wells were incubated and shacked for 15 minutes. Next, $20 \mu \mathrm{L}$ of lysate was transferred into a white 96-well plate, first: $20 \mu \mathrm{L}$ of Luciferase Assay regent II (Luc) was added to each well, the reading for the plate was done by using Tecan Spark-Luciferase program, second $20 \mu \mathrm{L}$ of Stop and Glo (S\&G) was added to each well and the reading for the plate was done by using Tecan Spark (Renilla program). 


\subsection{Results}

\subsubsection{Majority of human cancer cell lines tested arrest their cell cycle in growth-limiting conditions}

We are interested in understanding the key factors that drive $\mathrm{CT}$ genes activation in malignant cells. We hypothesize that loss-of-function of both cell cycle transcriptional repressor factors DREAM and Rb causes insensitivity to growth-limiting conditions. Therefore, we first aimed to identify human cancer cell lines that arrest versus cell lines that fail to arrest in limiting growth conditions, which would indicate that cell lines that loss of both DREAM and $\mathrm{Rb}$ functions cannot arrest in $\mathrm{G} 0 / \mathrm{G} 1$.

To categorize the response of human cancer cell lines to growth-limiting conditions, we measured the DNA content of the proliferated and serum-starved and serum released cells among ten cell lines. Using Propidium Iodide staining and flow cytometry, the DNA content of serum-starved cells was compared to normally proliferating and serum released cells. Serum starved cells were incubated in serum-free media for 48 hours, and serum released cells were serum-starved for 48 hours and then re-stimulated with media containing serum for 24 hours. We tested ten of human cancer cell lines, including breast cancer cell line (MDA-MB-231), human ovarian cancer cell line (ES-2), human ovarian carcinoma cell line (OVCAR-3), ovarian cancer cell line (OVCAR-5), glioblastoma cell line (T98G), glioblastoma cell line (8MGBA), colon 
adenocarcinoma cell line (SW480), lung cancer cell line (NCI-H1299), ovarian cancer cell line (SKOV3), and ovarian cancer cell line (OVCAR-10).

To assess whether cells were sensitive to serum starvation, we measured the percentages of total cells in G1/S (2n) and G2/M (4n) in serum-starved cells compared to normal proliferating cells (figure 4A and 4B), (figure 5A and 5B), (figure 6A and 6B), and (figure 7A and 7B). We found that 7 out of 10 cell lines were sensitive to growthlimiting conditions, indicating that they arrested in G0/G1. NC1-H1299 cells in G1/S increased from $55.65 \%$ to $89.70 \%$ in response to growth-limiting conditions, with a corresponding G2/M decrease from $29.30 \%$ to $4 \%$ (figure 4B). OVCAR-5 cells in G1/S increased from $65.57 \%$ to $83.87 \%$ in response to growth-limiting conditions, with a corresponding G2/M decrease from $20.51 \%$ to $8.13 \%$ (figure 4B). SW480 cells in G1/S increased from $65.57 \%$ to $74 \%$ in response to growth-limiting conditions, with a corresponding G2/M decrease from $18.49 \% \%$ to $8.01 \%$ (figure 4B). MDA-MB-231 cells in $\mathrm{G} 1 / \mathrm{S}$ increased from $63.30 \%$ to $81.80 \%$ in response to growth-limiting conditions, with a corresponding G2/M decrease from $25.40 \%$ to $13.30 \%$ (figure 5B). SKOV-3 cells in $\mathrm{G} 1 / \mathrm{S}$ increased from $52.67 \%$ to $76.73 \%$ in response to growth-limiting conditions, with a corresponding G2/M decrease from $28 \%$ to $14.57 \%$ (figure 5B). $8 \mathrm{MGBA}$ cells in G1/S increased from $64.50 \%$ to $84.30 \%$ in response to growth-limiting conditions, with a corresponding G2/M decrease from $22.53 \%$ to $9.97 \%$ (figure 5B). T98G cells in G1/S increased from $44.33 \%$ to $71.30 \%$ in response to growth-limiting conditions, with a corresponding G2/M decrease from $15.97 \%$ to $8.93 \%$ (figure $6 \mathrm{~B}$ ). These results indicate that NC1-H1299, OVCAR-5, SW480, MDA-MB-231, SKOV-3, 8MGBA, and T98G cell line arrested in G0/G1 in limiting growth conditions. 
In contrast, of the ten cell types, three cell lines were insensitive to growth conditions. ES-2 cells in G1/S and G2/M were 52-57\% and 33-35\% in all conditions (Figure 7B). OVCAR-3 cells in G1/S were $60-67 \%$, and G2/M were $18-25 \%$ in all conditions (Figure 7B). OVCAR-10 cells in G1/S were $51-52 \%$, and in G2/M, they were $26 \%$ in all conditions (Figure 7B). These results indicate that ES-2, OVCAR-3, and OVCAR-10 did not arrest in G0/G1 in limiting growth conditions.

In addition, we tested whether the repression of cell cycle genes during quiescence can be rescued by re-stimulation after serum starvation in OVCAR-5 cells that arrested in $\mathrm{G} 0 / \mathrm{G} 1$. As expected, the results displayed an increase in total cells in G1/S and G2/M upon re-stimulation (figure 4A). Upon re-stimulation, the cells re-entered the cell cycle and proliferated normally in G1/S and G2/M and show peaks in G1 and $\mathrm{G} 2 / \mathrm{M}$. The cell percentage in $\mathrm{G} 0 / \mathrm{G} 1$ is $65.57 \%$ and $20.51 \%$ in $\mathrm{G} 2 / \mathrm{M}$ in proliferated cells. While it is $83.87 \%$ in $\mathrm{G} 0 / \mathrm{G} 1$ and 8.13 in $\mathrm{G} 2 / \mathrm{M}$. However, it is $60.62 \%$ in $\mathrm{G} 0 / \mathrm{G} 1$ and $26.37 \%$ in $\mathrm{G} 2 / \mathrm{M}$ in released cells (figure 4B).

In total, 7 of cell lines arrested in response to serum starvation, including NC1H1299, OVCAR-5, SW480, MDA-MB-231, SKOV-3, 8MGBA, and T98G (figure 4A and 4B), (figure 5A and 5B) and (figure 6A and 6B). Three of the cell lines did not respond to serum starvation, including ES-2, OVCAR-3, and OVCAR-10 (figure 7A and 7B). 
Taking together, flow cytometry results revealed that the percentages of cells in G0/G1 and G2/M phases of the cell cycle significantly change in both proliferating and serum-starved cells in NC1-H1299, OVCAR-5, SW480, MDA-MB-231, SKOV-3, 8MGBA, and T98G cell lines because of the cell arrested in $\mathrm{G} 0 / \mathrm{G} 1$, and decreased in G2/M after serum starvation compared with proliferating cells. These cell lines that might have $\mathrm{Rb}$ or DREAM function correctly, are likely to be arrested in G0/G1 under growthlimiting conditions.

However, ES-2, OVCAR-3, and OVCAR-10 cell lines proliferated normally in G1, S, and G2/M even after serum starvation, and it might be as a result of loss activities of both DREAM complex and $\mathrm{Rb}$ that hinder cell cycle arrest following limiting growth conditions. These cell lines might lack both Rb and DREAM complex. Because of that, they are unable to exit the cell cycle. So, they proliferate normally. Thus, we are more interested in the cell lines that are arrested in G0/G1 (NC1-H1299, OVCAR-5, SW480, MDA-MB-231, SKOV-3, 8MGBA, and T98G to test how DREAM or $\mathrm{Rb}$ arrest cell cycle genes in $\mathrm{G} 0 / \mathrm{G} 1$ in response to limiting growth conditions and whether one of them is dysfunctional in these cell lines. 
A
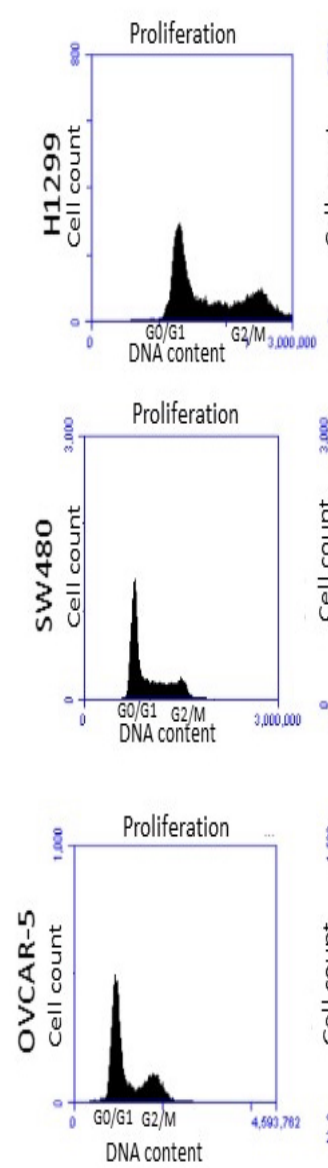
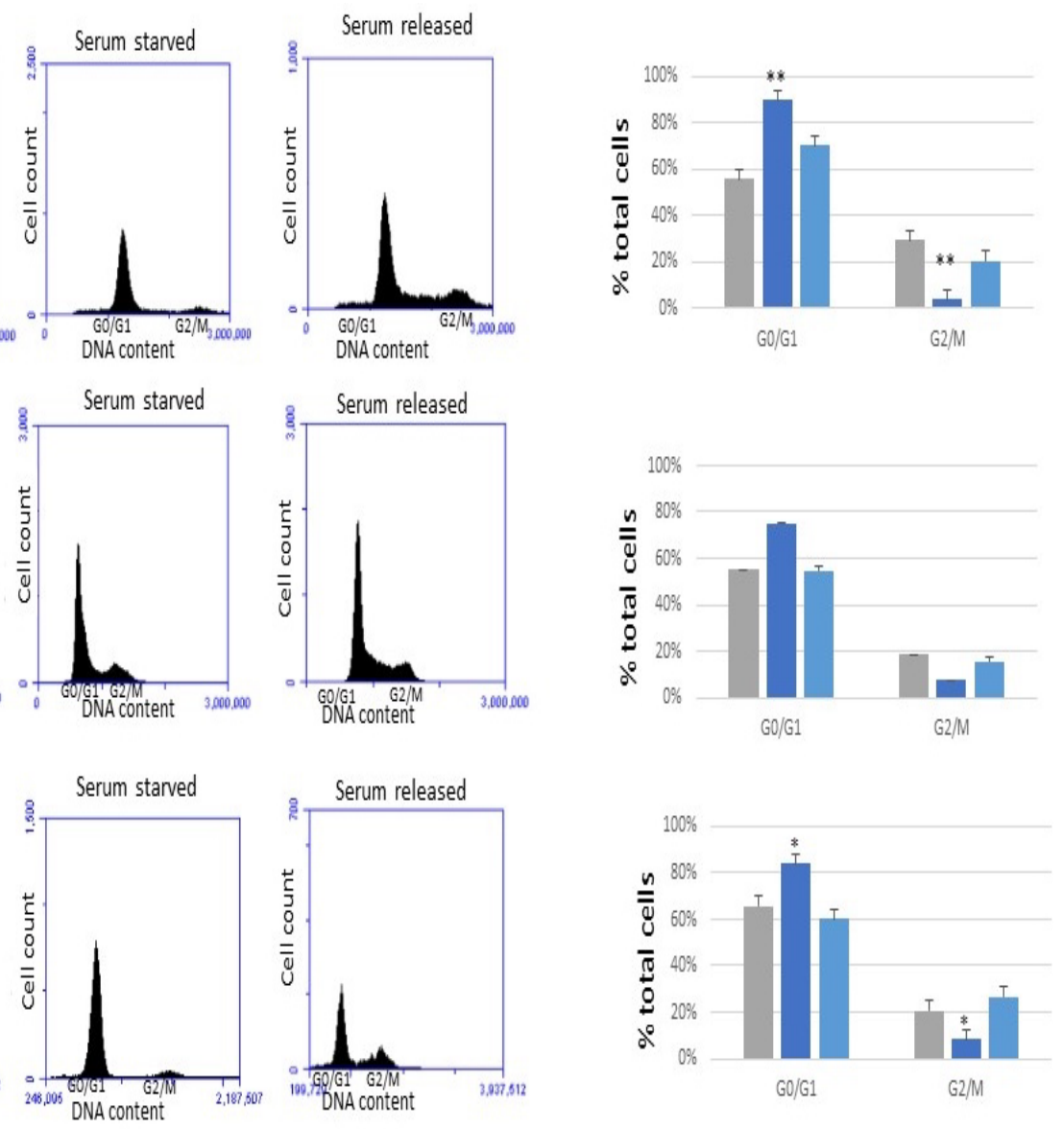

Figure 4 DNA content of the proliferating, serum-starved, and serum released cells of NCI-H1299, SW480, and OVCAR5 cell lines as measured by flow cytometry.

(A) In response to serum starvation, H1299, SW480, and OVCAR5 cell lines showed only one peak in G0/G1 phase indicating that they arrested in G0/G1.

(B) The total percentage of the cells of H1299, SW480, and OVCAR-5 cell lines are increased in G0/G1 phase and significantly decreased in G2/M phase in H1299, and OVCAR-5 after serum starvation for 48 hours. 
A
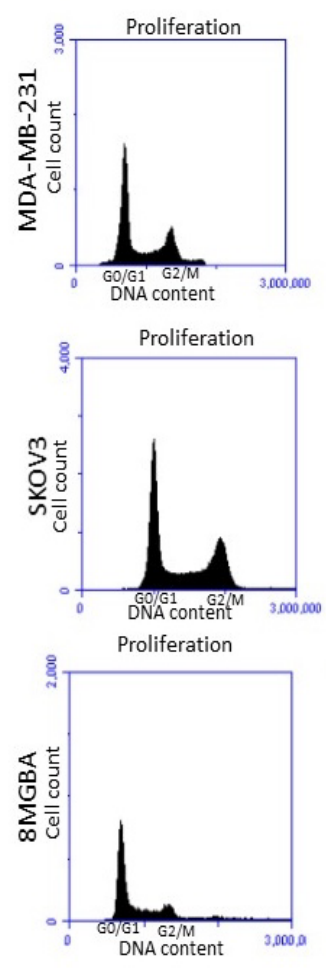
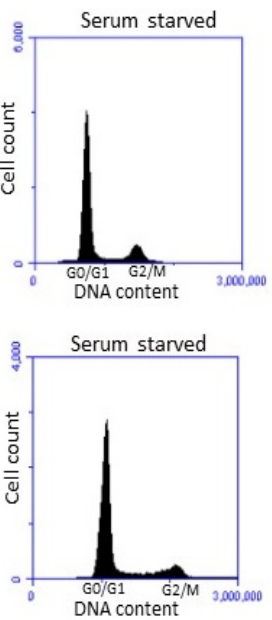

Serum starved

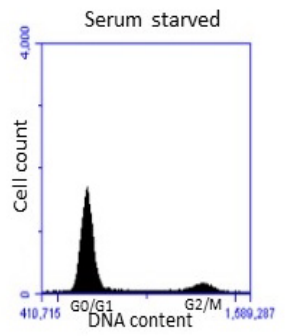

Serum released
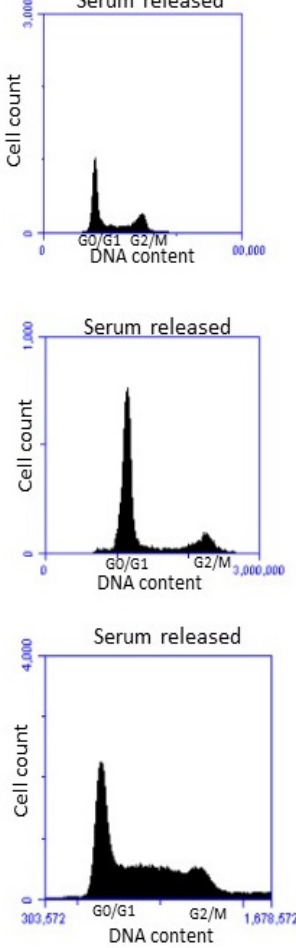

B
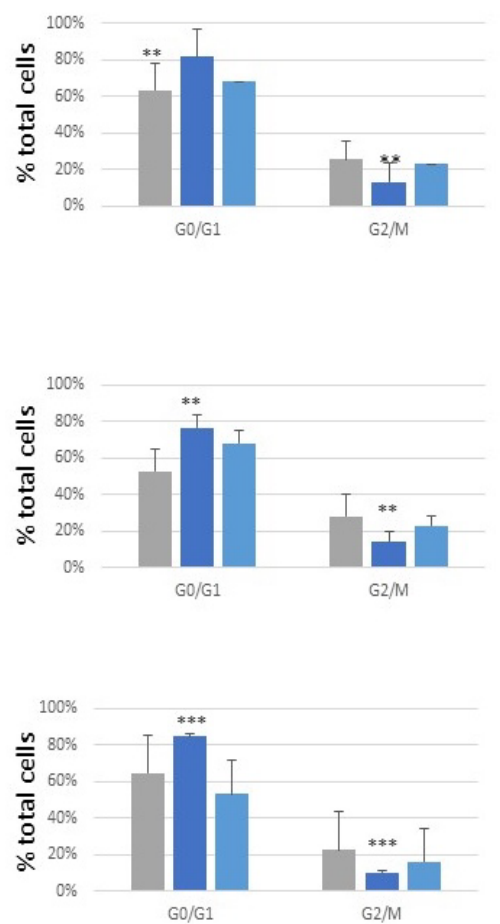

Figure 5 DNA content of the proliferating, serum-starved, and serum released cells of MDA-MB-231, SKOV3, and 8MGBA cell lines as measured by flow cytometry.

(A) In response to serum starvation, MDA-MB231, SKOV3, and 8MGBA cell lines show only one peak in G0/G1 phase indicating that they arrested in G0/G1.

(B) The total percentage of the cells of MDA-MB231, SKOV3, and 8MGBA cell lines are increased in G0/G1 phase and significantly decreased in G2/M phase after serum starvation for 48 hours. 
A

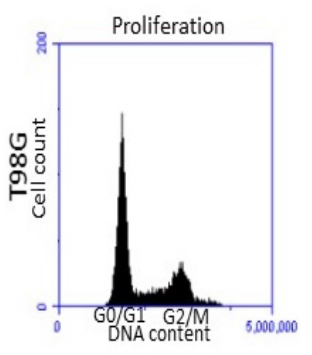

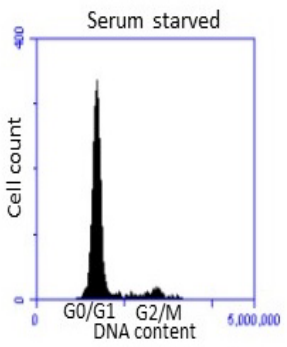

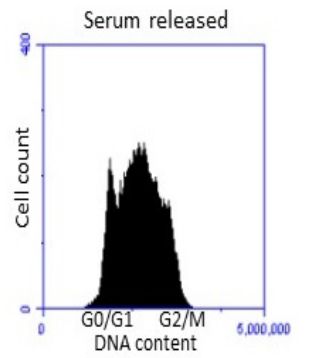

B

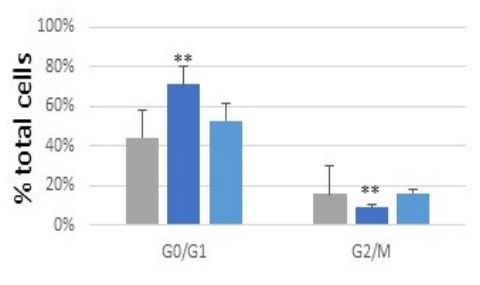

Figure 6 DNA content of the of the proliferating, serum-starved, and serum released cells $\mathrm{T} 98 \mathrm{G}$ cell line as measured by flow cytometry.

(A) In response to serum starvation, $\mathrm{T} 98 \mathrm{G}$ cell line showed only one peak in $\mathrm{G} 0 / \mathrm{G} 1$ phase indicating that it arrested in $\mathrm{G} 0 / \mathrm{G} 1$.

(B) The total percentage of the cells of T98G cell line are increased in G0/G1 and significantly decreased in G2/M phase after serum starvation for 48 hours. 
A
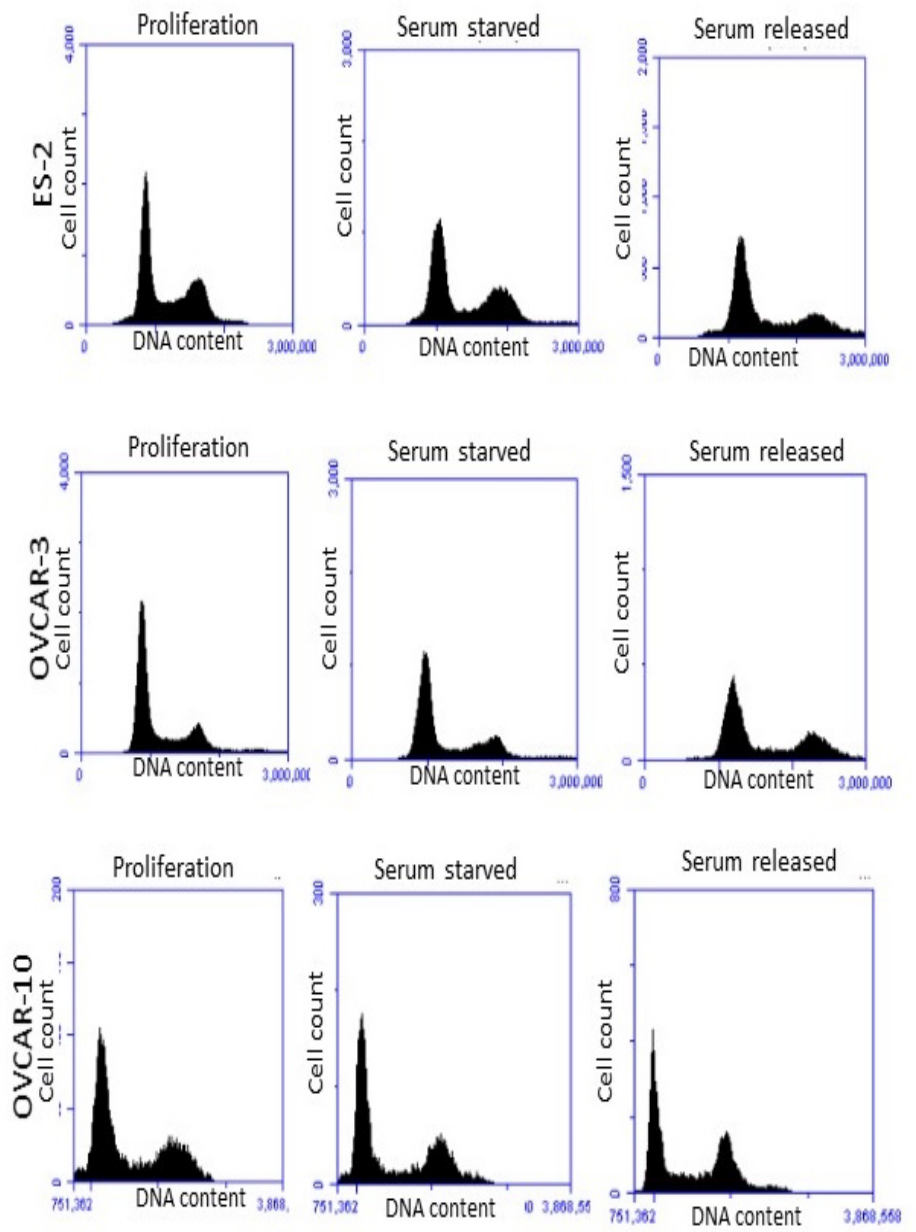

B
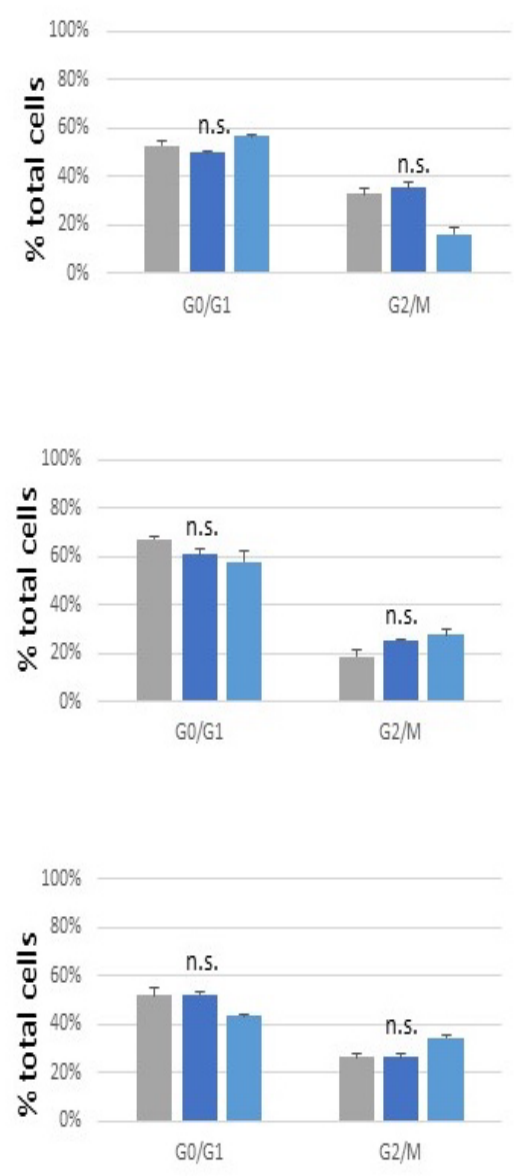

Figure 7 DNA content of the of the proliferating, serum-starved, and serum released cells of ES-2, OVCAR-3, and OVCAR-10 cell lines as measured by flow cytometry.

(A) ES-2, OVCAR-3, OVCAR-10 cell lines were insensitive to limiting growth conditions, and they show two peaks after serum starvation for 48 hours in $\mathrm{G} 1 / \mathrm{S}$ and $\mathrm{G} 2 / \mathrm{M}$ after serum starvation for 48 hours.

(B) The total percentage of the starved cells of ES-2, OVCAR-3, and OVCAR-10 did not significantly change in compared with proliferating and serum starved cells indicating that ES-2, OVCAR-3, and OVCAR-10 did not arrest in G0/G1 in limiting growth conditions. 


\subsubsection{Exploring a potential relationship between CT genes expression and loss of DREAM and Rb function}

If $\mathrm{CT}$ gene misexpression is linked to loss of DREAM and $\mathrm{Rb}$ function, then we expect that $\mathrm{CT}$ gene expression would be increased in cancer cells that are insensitive to serum starvation as compared to cells that arrest in growth-limiting conditions. We tested the mRNA expression of 12 known CT genes among ten cancer cell lines using reverse transcriptase quantitative PCR (RT-qPCR). Representative CT genes tested include MAGEA4, TTK, SSX2, LIN28B, PIWIL, CTAGE2, SKA3, MAGEA12, MAGEA3, MAGEA6, YBX2, and KCTD19 (Table 1.2).

CT genes are expressed in some human cancer cell lines that cannot arrest cells cycle in response to limiting growth conditions and proliferated normally in G1/S and G2/M. For example, MDA-MB3 cells expressed TTK and CTAGE2 genes (figure 8). OVCAR3 proliferated cells expressed TTK, SSX2, LIN28B, PIWIL, SKA3, YBX2, and CTAG2 genes (Figure 8) and (Figure 9). OVCAR10 cells expressed TTK, SSX2, and LIN28B (Figure 8). ES-2 expressed TTK and CTAG2 genes (Figure 8).

In addition, $\mathrm{CT}$ genes are expressed in some human cancer cell lines that can arrest in G0/G1 under limiting growth conditions. For instance, NCI-H1299 cells expressed MAGE4, TTK, and LIN28B genes (figure 8). SW480 cells expressed TTK, PIWIL, SKA3, YBX2, and KCTD19 genes (figure 8) and (figure 9). OVCAR5 and SKOV3 cells expressed TTK and CTAG2 genes (figure 8). 8MGBA cells expressed 
many CT genes such as TTK, SSX2, CTAG2, SKA3, MAGEA12, MAGEA3, MAGEA6, and KCTD19 (figure 8) and (figure 9). Finally, T98G expressed TTK and SKA3 genes (figure 8) and (figure 9).

Taking together, we did not find differences between two mRNA expression between the two groups. There are some cell lines that share similar CT genes expression patterns. For instance, TTK genes are expressed in all the cell lines that we tested. We discriminated between these two groups and select only cells that arrested in G0/G1 in limiting growth conditions. Because we did not observe differences of CT gene expression between cells that can arrest and cells that cannot. We next asked if misexpression of $\mathrm{CT}$ genes might be associated with dysfunction in either or both $\mathrm{Rb}$ or DREAM. 

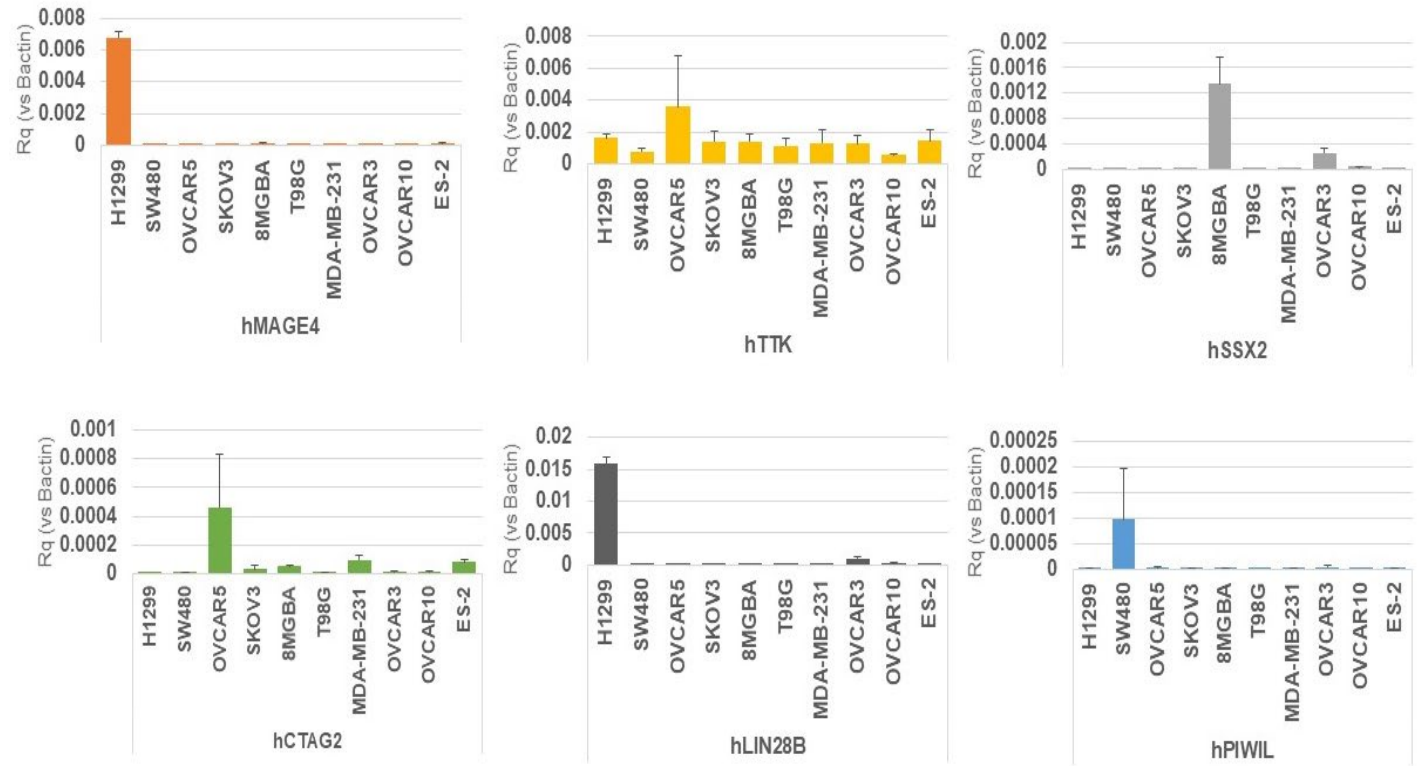

Figure 8 mRNA expression of CT genes in proliferation cells of H1299, SW480, OVCAR-5, SKOV3, 8MGBA, T98G, MDA-MB-231, OVCAR-3, OVCAR-10, and ES-2 cell lines was analyzed by $q$ PCR. 

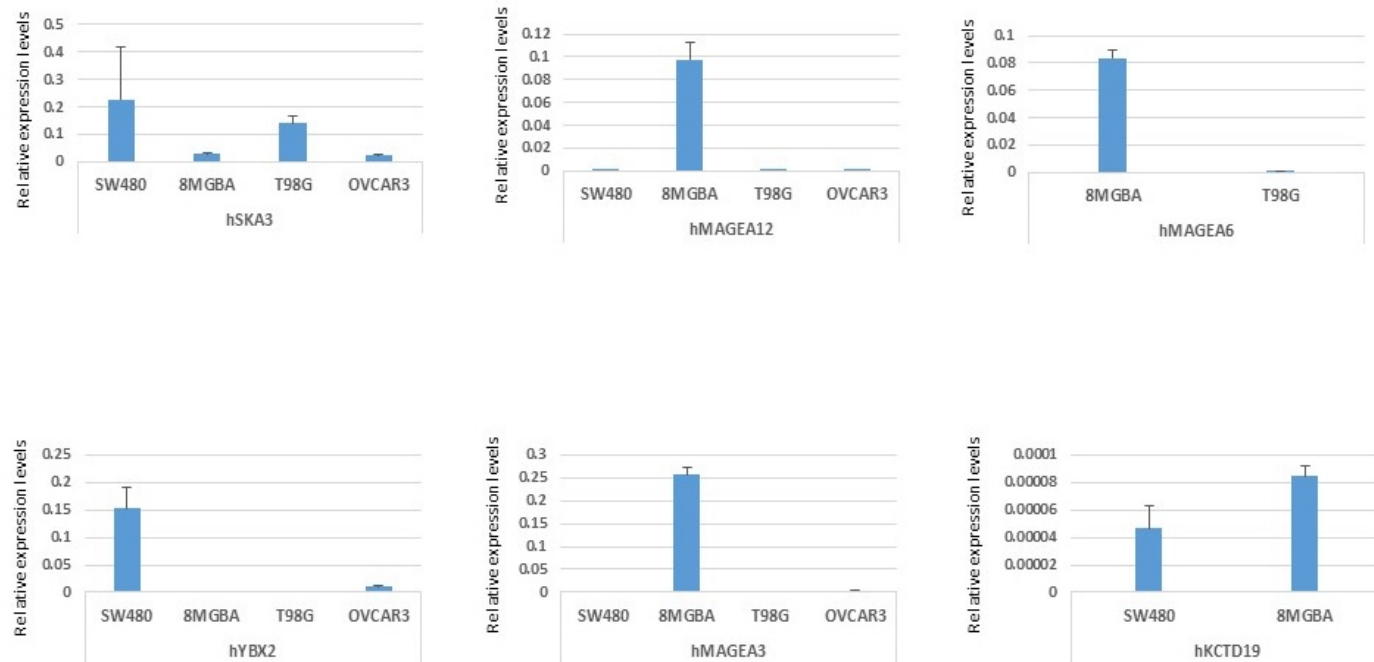

Figure 9 mRNA expression of CT genes in proliferation cells of SW480, 8MGBA, T98G, and OVCAR-3 cell lines was analyzed by $q$ PCR. 


\begin{tabular}{|c|c|c|c|c|c|c|c|c|c|}
\hline ACRV1 & ACTL9 & ASZ1 & BAGE2 & BEND2 & C17ORF64 & C8ORF74 & CABP2 & CABYR & CAPZA3 \\
\hline CAGE1 & CAPZA3 & CCDC155 & CATSPER4 & CCL27 & CTAG1B & DAZ1 & DBX1 & DCAF8L1 & DDI1 \\
\hline DEFB112 & DMRT1 & DNAJB3 & DPEP3 & DPPA3 & DSCR4 & DUSP21 & ENDOV & EPPIN & ERICH6 \\
\hline EXD1 & FABP12 & FAM187B & FAM209A & FAM221B & FAM46D & FAM71E1 & FAM9C & FATE1 & FBXO47 \\
\hline FKBP6 & FNDC8 & GAGE12D & GAGE12J & GGN & GJA8 & GOT1L1 & GPR32 & GSTA5 & GTSF1L \\
\hline H1FNT & HDGFL1 & HIST1H2BA & HIST1H2BB & HIST1H4F & HSFX2 & HYPM & IGLL1 & IL31 & IQCF6 \\
\hline IZUMO1 & IZUMO2 & KCNA10 & KCNU1 & KCNV2 & KIF24 & KLHL10 & KRT26 & KRT33A & LCA5L \\
\hline LCE2D & LCE3C & LELP1 & LIN28B & LRRC52 & MAEL & MAGEA1 & MAGEA12 & MAGEA2 & MAGEA3 \\
\hline MAGEA4 & MAGEA6 & MAGEA8 & MAGEA9B & MARCH11 & MMP20 & NANOS2 & NBPF6 & ODF1 & ODF4 \\
\hline OPN1LW & OPN5 & OR10J1 & OR13D1 & OR13G1 & OR14J1 & OR1C1 & OR1G1 & OR1K1 & OR1N1 \\
\hline OR2A4 & OR2D2 & OR2F1 & OR2G6 & OR2K2 & OR2W5 & OR4D1 & OR4E2 & OR52D1 & OR52E4 \\
\hline OR56A3 & OR5B2 & OR5B21 & OR5C1 & OR5H6 & OR5M11 & OR6C2 & OR6F1 & OR7E24 & OR8A1 \\
\hline OR8B2 & OR8B3 & OR8D1 & OTOP2 & PAPOLB & PCDHA1 & PCDHA13 & PDCL2 & PDILT & PDZD9 \\
\hline PFN4 & PGK2 & PHF7 & PIWIL3 & POM121L2 & POTEA & POTEG & POU5F2 & PP2D1 & PPEF2 \\
\hline PRAME & PRAMEF1 & PRKACG & PRM1 & PRM2 & PRPS1L1 & PRR23A & PRR23B & PRSS37 & PRSS41 \\
\hline PSG11 & PSG3 & PSKH2 & PSMA8 & PTF1A & R3HDML & RAD51AP2 & RBMXL2 & RBPJL & RGSL1 \\
\hline RIMBP3 & RIMBP3C & RNASE8 & RNF113B & RNF133 & RNF148 & ROPN1 & ROPN1B & S100A7L2 & SAGE1 \\
\hline SCN10A & SEL1L2 & SHCBP1L & SKA3 & SLC25A2 & SLC25A52 & SLCO6A1 & SLFNL1 & SMC1B & SMCP \\
\hline SOX30 & SPA17 & SPACA3 & SPATA12 & SPATA16 & SPATA17 & SPATA31D4 & SPATA33 & SPATA8 & SPINT3 \\
\hline SPRR4 & SSMEM1 & SSX1 & SSX2 & SUN3 & SUN5 & SYCE1L & SYCN & SYCP3 & SYNGR4 \\
\hline TAS2R1 & TCEB3C & TCP11 & TDRD12 & TEKT5 & TEX101 & TEX13B & TEX19 & TEX33 & TEX35 \\
\hline TEX38 & TEX43 & TMCO2 & TP53TG3B & TPPP2 & TPTE & TRIM48 & TSPAN16 & TXNDC2 & USP29 \\
\hline WBSCR 28 & WDR87 & WFDC10A & WFDC6 & YBX2 & ZAR1L & ZC2HC1B & ZDHHC19 & ZNF645 & ZSWIM2 \\
\hline
\end{tabular}

Table 1.2 Examples of cancer/testis genes (CT) 


\subsubsection{Early cell cycle genes are primarily repressed by both DREAM and Rb}

To test whether the DREAM complex or Rb are inactive in the cancer cell lines, we selected for further analyses SW480, NCI-H1299, and 8MGBA cells. We tested the mRNA expression of DREAM and Rb target genes in quiescent cells (serum-starved) and proliferated cells (serum-released) in SW480, NCI-H1299, and 8MGBA cell lines (Table 1.3). Our hypothesis suggests that cell lines that cannot arrest are dysfunctional in both $\mathrm{Rb}$ and $\mathrm{DREAM}$. However, cell lines that can arrest may be dysfunctional in either $\mathrm{Rb}$ or DREAM. Thus, we expect that either DREAM Complex or Rb work to repress CT genes in G0/G1 in the serum-starved cells in SW480, NCI-H1299, and 8MGBA starved cells. Therefore, one of them is broken.

We tested early cell cycle genes (G1/S), and late cell cycle genes (G2/M) using RT-qPCR analysis on serum-starved and serum released human cancer cell lines. We selected SW480, H1299, and 8MGBA cell lines.

To test the DREAM or Rb regulation of early cycle genes, we assessed expression levels of MCM5, ORC1, and CDC45 in quiescent cells (serum-starved) compared to proliferating cells (serum released). MCM5 has significantly downregulated in SW480 serum-starved cells compared with proliferating cells with 12 -fold change, suggesting that MCM5 gene is repressed by DREAM and Rb complexes in G0/G1 (figure 10). MCM5 is also downregulated in H1299 serum-starved cells with 3.8-fold repression and 
8MGBA with 2.7-fold repression (figure 10). However, the repression in H1299 and 8MGBA cell lines is not significant. Similarly, ORC1 is also significant downregulated in SW480 serum-starved cells compared with proliferating cells with the 7.5 -fold change, and it is downregulated in H1299 starved- cells with 2.2 fold change compared to proliferated cells. It is also downregulated in 8MGBA with 2.4 fold change (figure 10). The expression of the CDC45 gene is lowly expressed in SW480 starved-cells with 3.6 compared to proliferating cells (figure 10). In addition, it is lowly expressed in H1299 serum-starved cells with a 6.7-fold change and downregulated in serum-starved 8MGBA with a 3.4-fold change (figure 10).

Taken together, these results testing early cell cycle genes in NCI-H1299 and SW480 and 8MGBA cell lines suggests that either DREAM or Rb are functional. However, the lower fold change observed in NCI-H1299 and 8MGBA serum-starved cells as compared to proliferating cells suggest that 1 of the factors may be dysfunctional. 

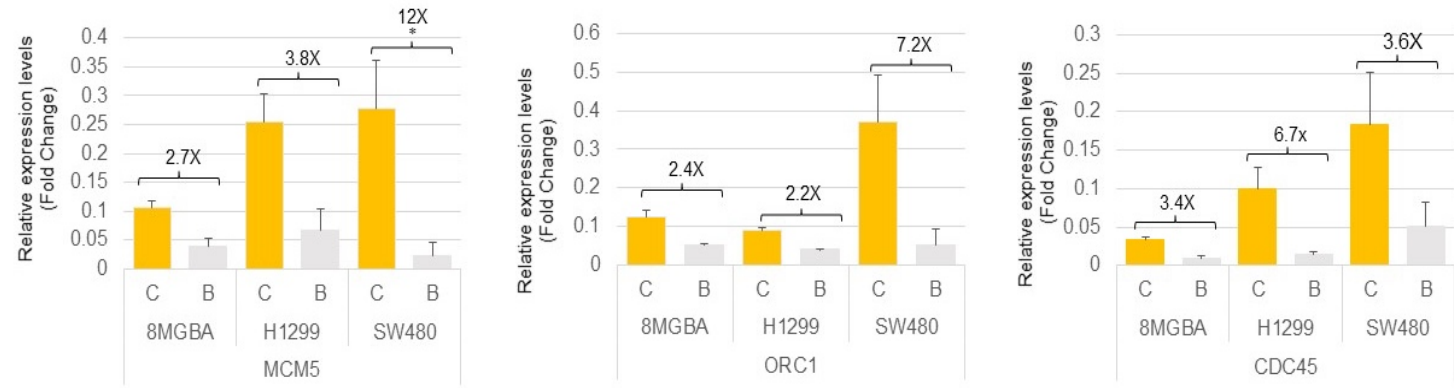

Figure 10 mRNA expression of early cell cycle genes (MCM5, ORC1, and CDC45) in the proliferated cells (yellow) and the quiescent cells (gray) in 8MGBA, H1299, SW480 cell lines was measured by qPCR and normalized to U6 expression. The mRNA expression results suggest that DREAM and Rb G0/G1 target genes are still down-regulated in serum-starved cells as compared to proliferating cells. 


\subsubsection{Late cell cycle genes are primarily repressed by DREAM}

Since the only DREAM represses late cell cycle genes, we assess expression levels of CCNB2, PBK, and BUB1 in quiescent cells (serum-starved) and proliferating cells (serum released). The expression of CCNB2 gene is downregulated in SW480 cells with 1.7 fold changes and NCI-H1299 with 1.3 fold changes and 8MGBA starved cells with 4 fold changes (figure 11). The expression of the PBK gene is partially repressed in SW480 cells with 2 fold change, and it is lowly expressed in H1299 quiescent cells with 3.9 fold change, and in 8MGBA starved cells with 5.8 fold change (figure 11). The expression of the BUB1 gene is substantially impaired in SW480 serum-starved cells with 1 fold change (figure 11). It is partially downregulated in H1299 quiescent cells with fold change 4.9 (Figure 11), and it is downregulated in 8MGBA with fold change 5.9 (figure 11)

Taken together, these results testing late cell cycle genes in NCI-H1299 and SW480 and 8MGBA cell lines suggest that DREAM may be dysfunctional in NCI-1299 and SW490, but the results are ambiguous. 

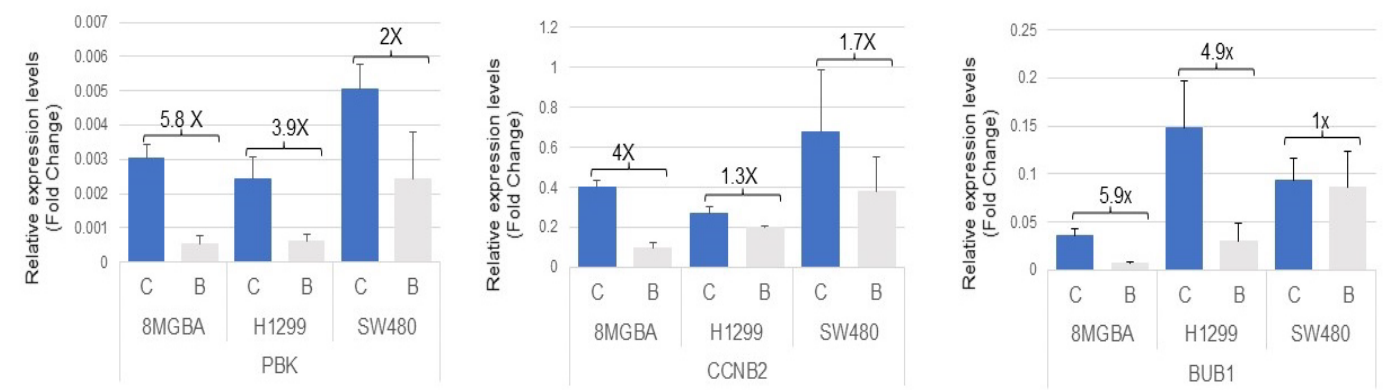

Figure 11 mRNA expression of late cell cycle genes (CCNB2, PBK, and BUB1) in the proliferated cells (blue) and the quiescent cells (gray) in 8MGBA, H1299, SW480 cell lines was measured by qPCR and normalized to U6 expression. Analysis of G2/M late cell cycle genes expression in serum starved cells suggest that DREAM is dysfunctional in SW480 and H1299 cell lines. 


\begin{tabular}{|c|c|c|}
\hline Gene name & $\begin{array}{l}\text { Litovchick } \\
2007 \\
\text { DREAM }\end{array}$ & $\begin{array}{c}\text { Chicas } 2010 \\
\text { RB }\end{array}$ \\
\hline ANLN (Anilin) & + & + \\
\hline AURKA (AIK-1) & + & + \\
\hline AURKB (AIM-1) & + & - \\
\hline BIRC5 (Survivin) & + & - \\
\hline BRCA1 & + & + \\
\hline BUB1 & + & - \\
\hline BUB1B (BUBR1) & + & - \\
\hline CCNA1 & + & - \\
\hline CCNA2 & + & - \\
\hline CCNB1 & + & - \\
\hline CCNB2 & + & - \\
\hline CDC20 & + & - \\
\hline CDC25A & + & + \\
\hline CDC25B & + & - \\
\hline CDC25C & + & - \\
\hline CDC45 & + & + \\
\hline CDC6 & + & + \\
\hline CDK1 (CDC2) & + & + \\
\hline CENPA & + & - \\
\hline CENPE & + & + \\
\hline
\end{tabular}




\begin{tabular}{|c|c|c|}
\hline CENPF & + & + \\
\hline CHAF1B & + & - \\
\hline CHEK1 (CHK1) & + & + \\
\hline $\mathrm{CIT}$ & + & + \\
\hline CKS1B (CKS1) & + & + \\
\hline CKS2 & + & + \\
\hline DCK & + & + \\
\hline DDX11 (CHLR1) & + & + \\
\hline DHFR & + & + \\
\hline DLGAP5 & + & + \\
\hline E2F1 & + & + \\
\hline E2F5 & + & - \\
\hline E2F8 & + & + \\
\hline ЕСТ2 & + & - \\
\hline $\mathrm{EZH} 2$ & + & - \\
\hline EXO1 & + & + \\
\hline FANCG & + & + \\
\hline FEN1 & + & + \\
\hline FOXM1 (MPP2) & + & + \\
\hline GJB2 & + & - \\
\hline HMGB1 & + & + \\
\hline HMGB2 & + & + \\
\hline ING1 & + & + \\
\hline
\end{tabular}




\begin{tabular}{|l|r|r|}
\hline KI67 (MKI67) & + & + \\
KIF2C (MCAK) & + & + \\
KIF4A & + & + \\
KIF11 (KNSL1) & + & + \\
KIF23 (KNSL5) & + & + \\
KIFC1 (HSET) & + & + \\
LIG1 & + & + \\
MAD2L1 (MAD2) & + & + \\
MCM2 & + & + \\
MCM3 & + & + \\
MCM4 & + & + \\
PLK1 (Sak-b) & + & + \\
MCM5 & + & + \\
MCM6 & + & + \\
MCM7 & + & + \\
NCAPH (C-mYC & + & + \\
\hline
\end{tabular}




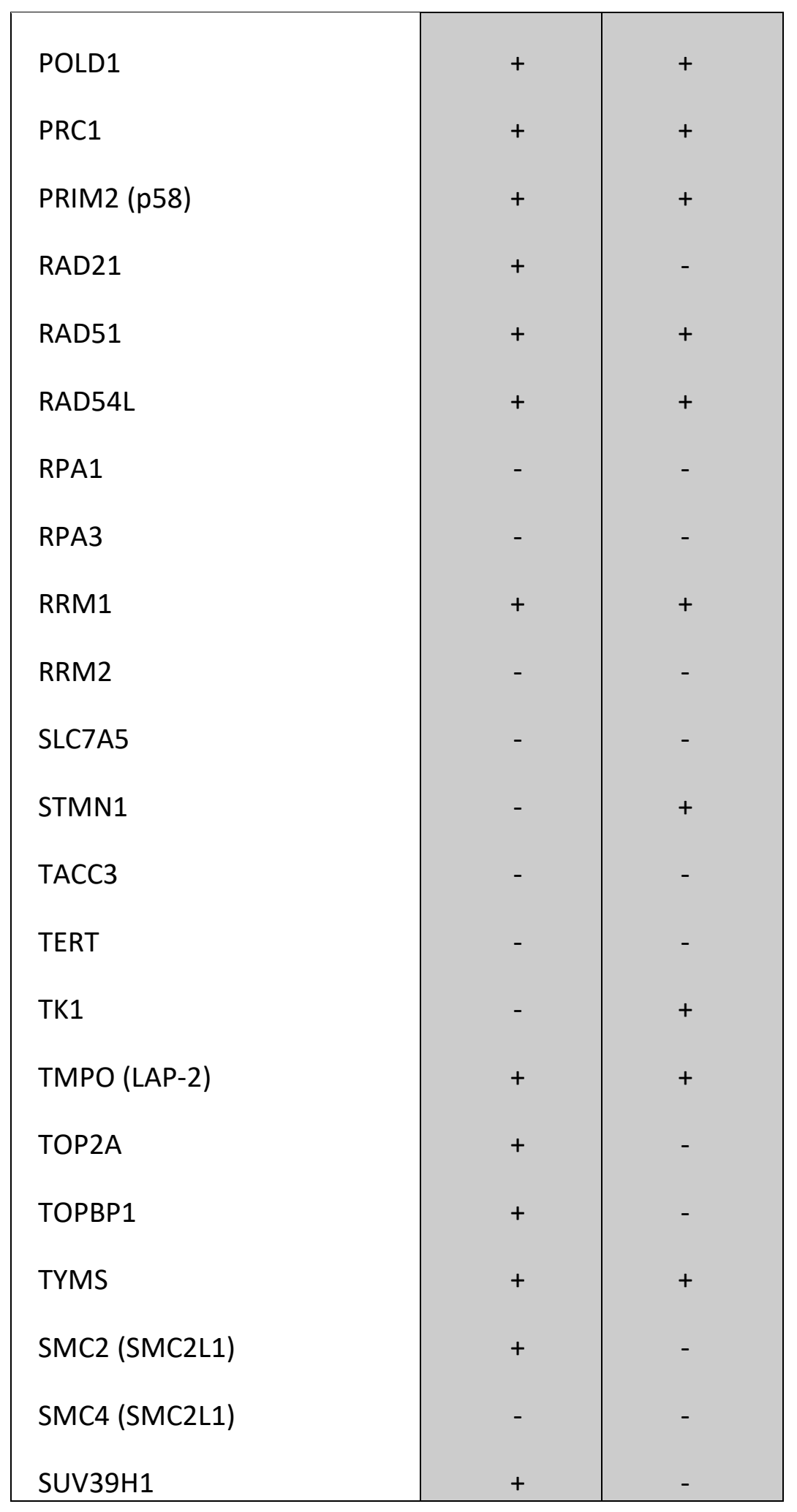

Table 1.3 Examples of DREAM and Rb target genes 


\subsubsection{DREAM is functional normally in SW480, and $\mathrm{H} 1299$ cell lines}

To test whether DREAM or Rb are functioning in the SW480 and H1299 cell lines, we performed a luciferase reporter assay. We tested DREAM and RB functions in quiescent cells (starved cells) and whether either one of them works or not. In SW480 serum-starved cells, we did not observe an increase in activity of the ORC1 promoter when the E2F binding site is mutated (ORC1 E2F) as compared to the wild-type ORC1 promoter (ORC1 WT). This might reflect to loss of function of the $\mathrm{Rb}$ (Figure 12). In contrast, the wild-type ORC1 promoter is repressed compared to ORC1 E2F, suggesting that $\mathrm{Rb}$ is functional. A small increase the activity of Bub1 promoter when the CHR binding site is mutated (Bub1 CHR) as compared to Bub1 WT suggests that DREAM is functional in SW480 and NCI H1299 (Figure 12).

Taken together, the results of the luciferase assay suggest that $\mathrm{Rb}$ is disfunctional in SW480, but results for DREAM are ambigous compared to the late cell cycle gene expression analysis (Figure 12). 

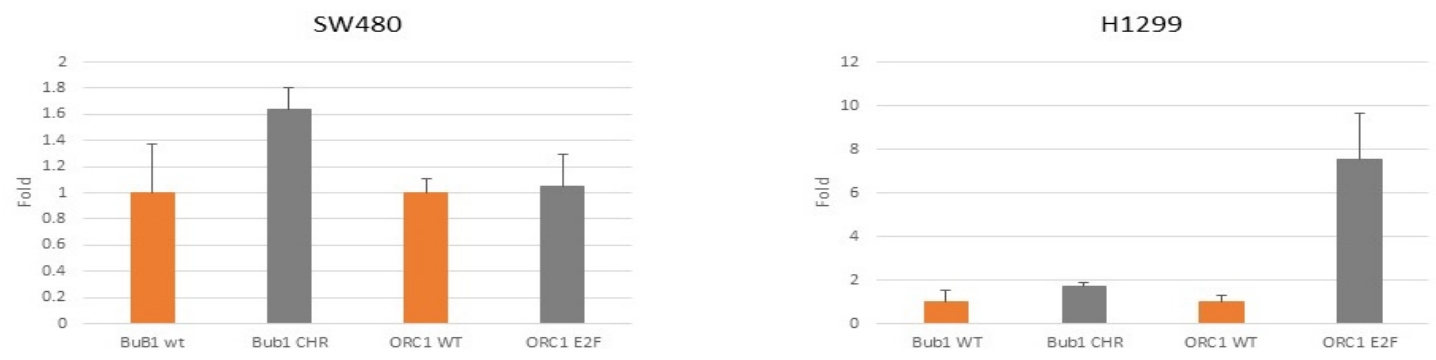

Figure 12 The activity of E2F binding sites (Orc1) and CHR binding sites (Bub1) in the wild type (WT) (orange) and the serum-starved cells (grey) in the SW480 and H1299 cell lines were analyzed by luciferase reporter assay. 


\subsection{Discussion}

Previous studies reported that the Cancer/testis genes (CT) are ectopically expressed in various types of human cancers. They are expressed in bladder, lung, ovarian, melanoma, and breast tumors [36]. These genes are exclusively expressed in male germ cells and placenta [39]. The factors that drive the misexpression of CT genes that impacts somatic cells expression and promote the development of cancer remain poorly understood. Our study hypothesized that misexpression of CT genes in cancer cells is associated with dysfunction of both the transcription suppressors DREAM complex and $\mathrm{Rb}$ that regulate cell cycle genes through the p53-DREAM pathway.

The flow cytometry results revealed that serum-starvation induced cancer cells to arrest in G0/G1 in respond to limiting growth conditions. Thus, the total percent of the cells decreased in S, G2, and M phase indicating that these cell lines arrested in the G0/G1 phase and Rb or DREAM function as normal (figure 4A, and 4B), (figure 5A and 5B), and (figure 6A and 6B). However, the cell lines that were insensitive to serum starvation might have a loss of functions of both DREAM and RB (figure 7A, and 7B).

Furthermore, one of the unexpected results that gene expression analysis of all CT genes revealed that they have similar gene expression patterns indicating that we did not find a difference in gene expression between cells that can arrest and the cells that cannot in limiting growth conditions (figure 8) and (figure 9). Thus, maybe we should test more 
CT genes to find an appropriate CT with specific expression pattern that is expressed only in cancer cells that are lacking both DREAM and $\mathrm{Rb}$ and cannot exit cell cycle. Moreover, gene expression analysis of early cell cycle genes G0/G1 (MCM5, ORC1, and CDC45), and late cell cycle genes G2/M (PBK, BUB1, CCNB2) (figure 10) and (figure 11) revealed that that either the DREAM or RB is dysfunction in $\mathrm{G} 0 / \mathrm{G} 1$. The gene expression analysis of late cell cycle genes (CCNB2, PBK, and BUB1) revealed unexpected results that suggest that DREAM is dysfunctional in SW480 and H1299 cell lines.

Furthermore, to test more directly whether DREAM or $\mathrm{Rb}$ is dysfunctional in SW480 and H1299, we performed a Luciferase Reporter assay. In contrast to the mRNA gene expression results, the results revealed that Rb may be dysfunctional in SW480 cells (figure 12). Together, these results do not confirm that loss of DREAM or Rb is linked to activation of $\mathrm{CT}$ gene expression in human cancer cell lines. 


\subsection{Conclusion}

Our study indicates the critical role of DREAM complex in regulating cell cycle genes in early cell cycle phases G0, G1 by binding to E2F or E2F and CLE sites, and late cell cycle genes $\mathrm{G} 2 / \mathrm{M}$ by binding to $\mathrm{CHR}$ or $\mathrm{CHR}$ and $\mathrm{CDE}$ sites. It remains unclear how the molecular functions of the DREAM complex differ from $\mathrm{Rb}$. How do they work together or overlap to repress cell cycle genes? It is unclear how p53 influences the P53DREAM pathway to repress cell cycle in $\mathrm{G} 0 / \mathrm{G} 1$ and $\mathrm{G} 2 / \mathrm{M}$ or if other unknown genes have similar functions to repress or active cell cycle genes. However, in order to test p53 function, additional studies such as western plot in p53 knockout cells have to be conducted. Despite these results, much remains to be learned about how DREAM complex and the pocket proteins regulate cell cycle genes through the P53-DREAM pathway. In particular, concerning their roles in the soma-to-germline transformation that could be associated with cancer development in human mammalian cells.

Although unexpected results, our study may provide an insight into the functions of DREAM complex and Rb as transcriptional repressors through the cell cycle genes, and how dysfunction of both of them might contribute to promoting tumors development. 


\subsection{References}

1. Sharma, S., T.K. Kelly, and P.A. Jones, Epigenetics in cancer. Carcinogenesis, 2010. 31(1): p. 27-36.

2. Weir, H.K., et al., The past, present, and future of cancer incidence in the United States: 1975 through 2020. Cancer, 2015. 121(11): p. 1827-37.

3. Hanahan, D. and R.A. Weinberg, Hallmarks of cancer: the next generation. Cell, 2011. 144(5): p. 646-74.

4. Dick, F.A. and S.M. Rubin, Molecular mechanisms underlying RB protein function. Nat Rev Mol Cell Biol, 2013. 14(5): p. 297-306.

5. Macaluso, M., et al., Modulation of cell cycle components by epigenetic and genetic events. Semin Oncol, 2005. 32(5): p. 452-7.

6. Sadasivam, S. and J.A. DeCaprio, The DREAM complex: master coordinator of cell cycle-dependent gene expression. Nat Rev Cancer, 2013. 13(8): p. 585-95.

7. Engeland, K., Cell cycle arrest through indirect transcriptional repression by p53: I have a DREAM. Cell Death Differ, 2018. 25(1): p. 114-132.

8. Funk, L.C., L.M. Zasadil, and B.A. Weaver, Living in CIN: Mitotic Infidelity and Its Consequences for Tumor Promotion and Suppression. Dev Cell, 2016. 39(6): p. 638-652.

9. Scanlan, M.J., A.J. Simpson, and L.J. Old, The cancer/testis genes: review, standardization, and commentary. Cancer Immun, 2004. 4: p. 1.

10. Sever, R. and J.S. Brugge, Signal transduction in cancer. Cold Spring Harb Perspect Med, 2015. 5(4).

11. Hanahan, D. and R.A. Weinberg, The hallmarks of cancer. Cell, 2000. 100(1): p. 57-70.

12. Engel, B.E., W.D. Cress, and P.G. Santiago-Cardona, The Retinoblastoma Protein: A Master Tumor Suppressor Acts as a Link between Cell Cycle and Cell Adhesion. Cell Health Cytoskelet, 2015. 7: p. 1-10.

13. Egger, J.V., et al., Dephosphorylation of the Retinoblastoma protein (Rb) inhibits cancer cell EMT via Zeb. Cancer Biol Ther, 2016. 17(11): p. 1197-1205.

14. Tower, J., Programmed cell death in aging. Ageing Res Rev, 2015. 23(Pt A): p. 90-100.

15. Chen, J., The Cell-Cycle Arrest and Apoptotic Functions of 533 in Tumor Initiation and Progression. Cold Spring Harb Perspect Med, 2016. 6(3): p. a026104.

16. Campisi, J., Aging, cellular senescence, and cancer. Annu Rev Physiol, 2013. 75: p. 685-705.

17. Ozaki, T. and A. Nakagawara, Role of p53 in Cell Death and Human Cancers. Cancers (Basel), 2011. 3(1): p. 994-1013.

18. Amin, A., et al., Evasion of anti-growth signaling: A key step in tumorigenesis and potential target for treatment and prophylaxis by natural compounds. Semin Cancer Biol, 2015. 35 Suppl: p. S55-S77.

19. Sherr, C.J. and F. McCormick, The RB and p53 pathways in cancer. Cancer Cell, 2002. 2(2): p. 103-12. 
20. Elmore, S., Apoptosis: a review of programmed cell death. Toxicol Pathol, 2007. 35(4): p. 495-516.

21. Igney, F.H. and P.H. Krammer, Death and anti-death: tumour resistance to apoptosis. Nat Rev Cancer, 2002. 2(4): p. 277-88.

22. Campbell, K.J. and S.W.G. Tait, Targeting BCL-2 regulated apoptosis in cancer. Open Biol, 2018. 8(5).

23. Blackburn, E.H. and K. Collins, Telomerase: an RNP enzyme synthesizes DNA. Cold Spring Harb Perspect Biol, 2011. 3(5).

24. Jafri, M.A., et al., Roles of telomeres and telomerase in cancer, and advances in telomerase-targeted therapies. Genome Med, 2016. 8(1): p. 69.

25. Hahn, W.C. and M. Meyerson, Telomerase activation, cellular immortalization and cancer. Ann Med, 2001. 33(2): p. 123-9.

26. Cong, Y.S., W.E. Wright, and J.W. Shay, Human telomerase and its regulation. Microbiol Mol Biol Rev, 2002. 66(3): p. 407-25, table of contents.

27. Akincilar, S.C., B. Unal, and V. Tergaonkar, Reactivation of telomerase in cancer. Cell Mol Life Sci, 2016. 73(8): p. 1659-70.

28. Nishida, N., et al., Angiogenesis in cancer. Vasc Health Risk Manag, 2006. 2(3): p. 213-9.

29. Gasparini, G., et al., Tumor microvessel density, p53 expression, tumor size, and peritumoral lymphatic vessel invasion are relevant prognostic markers in nodenegative breast carcinoma. J Clin Oncol, 1994. 12(3): p. 454-66.

30. Sigismund, S., D. Avanzato, and L. Lanzetti, Emerging functions of the EGFR in cancer. Mol Oncol, 2018. 12(1): p. 3-20.

31. Radinsky, R., et al., Level and function of epidermal growth factor receptor predict the metastatic potential of human colon carcinoma cells. Clin Cancer Res, 1995. 1(1): p. 19-31.

32. Wells, A., et al., Targeting tumor cell motility as a strategy against invasion and metastasis. Trends Pharmacol Sci, 2013. 34(5): p. 283-9.

33. Xie, K., et al., Cancer-testis antigens in ovarian cancer: implication for biomarkers and therapeutic targets. J Ovarian Res, 2019. 12(1): p. 1.

34. Gurchot, C., The trophoblast theory of cancer (John Beard, 1857-1924) revisited. Oncology, 1975. 31(5-6): p. 310-33.

35. Turco, M.Y., et al., Trophoblast organoids as a model for maternal-fetal interactions during human placentation. Nature, 2018. 564(7735): p. 263-267.

36. Simpson, A.J., et al., Cancer/testis antigens, gametogenesis and cancer. Nat Rev Cancer, 2005. 5(8): p. 615-25.

37. Old, L.J., Cancer/testis (CT) antigens - a new link between gametogenesis and cancer. Cancer Immun, 2001. 1: p. 1.

38. Strome, S. and D. Updike, Specifying and protecting germ cell fate. Nat Rev Mol Cell Biol, 2015. 16(7): p. 406-16.

39. Scanlan, M.J., et al., Cancer/testis antigens: an expanding family of targets for cancer immunotherapy. Immunol Rev, 2002. 188: p. 22-32.

40. Rechtsteiner, A., et al., Repression of Germline Genes in Caenorhabditis elegans Somatic Tissues by H3K9 Dimethylation of Their Promoters. Genetics, 2019. 212(1): p. 125-140. 
41. Ross, M.T., et al., The DNA sequence of the human X chromosome. Nature, 2005. 434(7031): p. 325-37.

42. Hofmann, O., et al., Genome-wide analysis of cancer/testis gene expression. Proc Natl Acad Sci U S A, 2008. 105(51): p. 20422-7.

43. Caballero, O.L. and Y.T. Chen, Cancer/testis (CT) antigens: potential targets for immunotherapy. Cancer Sci, 2009. 100(11): p. 2014-21.

44. Yao, J., et al., Tumor subtype-specific cancer-testis antigens as potential biomarkers and immunotherapeutic targets for cancers. Cancer Immunol Res, 2014. 2(4): p. 371-9.

45. Gjerstorff, M.F., M.H. Andersen, and H.J. Ditzel, Oncogenic cancer/testis antigens: prime candidates for immunotherapy. Oncotarget, 2015. 6(18): $\mathrm{p}$. 15772-87.

46. Taguchi, A., et al., A search for novel cancer/testis antigens in lung cancer identifies $V C X / Y$ genes, expanding the repertoire of potential immunotherapeutic targets. Cancer Res, 2014. 74(17): p. 4694-705.

47. Wu, Y., M. Sarkissyan, and J.V. Vadgama, Epithelial-Mesenchymal Transition and Breast Cancer. J Clin Med, 2016. 5(2).

48. Wang, D., et al., Somatic misexpression of germline P granules and enhanced $R N A$ interference in retinoblastoma pathway mutants. Nature, 2005. 436(7050): p. 593-7.

49. Pothof, J., et al., Identification of genes that protect the C. elegans genome against mutations by genome-wide RNAi. Genes Dev, 2003. 17(4): p. 443-8.

50. van den Heuvel, S. and N.J. Dyson, Conserved functions of the $p R B$ and E2F families. Nat Rev Mol Cell Biol, 2008. 9(9): p. 713-24.

51. Cobrinik, D., Pocket proteins and cell cycle control. Oncogene, 2005. 24(17): p. 2796-809.

52. Bertoli, C., J.M. Skotheim, and R.A. de Bruin, Control of cell cycle transcription during G1 and S phases. Nat Rev Mol Cell Biol, 2013. 14(8): p. 518-28.

53. Wikenheiser-Brokamp, K.A., Retinoblastoma regulatory pathway in lung cancer. Curr Mol Med, 2006. 6(7): p. 783-93.

54. Liu, H., et al., New roles for the RB tumor suppressor protein. Curr Opin Genet Dev, 2004. 14(1): p. 55-64.

55. Knudsen, E.S. and J.Y. Wang, Targeting the RB-pathway in cancer therapy. Clin Cancer Res, 2010. 16(4): p. 1094-9.

56. Narasimha, A.M., et al., Cyclin D activates the Rb tumor suppressor by monophosphorylation. Elife, 2014. 3.

57. Yeo-Teh, N.S.L., Y. Ito, and S. Jha, High-Risk Human Papillomaviral Oncogenes E6 and E7 Target Key Cellular Pathways to Achieve Oncogenesis. Int J Mol Sci, 2018. 19(6).

58. Litovchick, L., et al., Evolutionarily conserved multisubunit RBL2/p130 and E2F4 protein complex represses human cell cycle-dependent genes in quiescence. Mol Cell, 2007. 26(4): p. 539-51.

59. Vousden, K.H. and C. Prives, Blinded by the Light: The Growing Complexity of p53. Cell, 2009. 137(3): p. 413-31. 
60. Bohlig, L. and K. Rother, One function--multiple mechanisms: the manifold activities of $p 53$ as a transcriptional repressor. J Biomed Biotechnol, 2011. 2011: p. 464916.

61. Soussi, T., et al., Meta-analysis of the $p 53$ mutation database for mutant p53 biological activity reveals a methodologic bias in mutation detection. Clin Cancer Res, 2006. 12(1): p. 62-9.

62. Weisz, L., M. Oren, and V. Rotter, Transcription regulation by mutant $p 53$. Oncogene, 2007. 26(15): p. 2202-11.

63. Peart, M.J. and C. Prives, Mutant 533 gain of function: the NF-Y connection. Cancer Cell, 2006. 10(3): p. 173-4.

64. Wade, M., Y.C. Li, and G.M. Wahl, MDM2, MDMX and p53 in oncogenesis and cancer therapy. Nat Rev Cancer, 2013. 13(2): p. 83-96.

65. Kruse, J.P. and W. Gu, Modes of p53 regulation. Cell, 2009. 137(4): p. 609-22.

66. Riley, T., et al., Transcriptional control of human p53-regulated genes. Nat Rev Mol Cell Biol, 2008. 9(5): p. 402-12.

67. Sullivan, K.D., et al., Mechanisms of transcriptional regulation by $p 53$. Cell Death Differ, 2018. 25(1): p. 133-143.

68. Schmit, F., et al., LINC, a human complex that is related to $p R B$-containing complexes in invertebrates regulates the expression of G2/M genes. Cell Cycle, 2007. 6(15): p. 1903-13.

69. Fischer, M., et al., Integration of TP53, DREAM, MMB-FOXM1 and RB-E2F target gene analyses identifies cell cycle gene regulatory networks. Nucleic Acids Res, 2016. 44(13): p. 6070-86.

70. Muller, G.A., et al., Timing of transcription during the cell cycle: Protein complexes binding to E2F, E2F/CLE, CDE/CHR, or CHR promoter elements define early and late cell cycle gene expression. Oncotarget, 2017. 8(58): p. 97736-97748.

71. Fischer, M. and G.A. Muller, Cell cycle transcription control: DREAM/MuvB and RB-E2F complexes. Crit Rev Biochem Mol Biol, 2017. 52(6): p. 638-662.

72. Musa, J., et al., MYBL2 (B-Myb): a central regulator of cell proliferation, cell survival and differentiation involved in tumorigenesis. Cell Death Dis, 2017. 8(6): p. e2895. 